\title{
GENERALIZED RESOLVENTS OF ORDINARY DIFFERENTIAL OPERATORS $\left({ }^{1}\right)$
}

\author{
BY \\ EARL A. CODDINGTON AND RICHARD C. GILBERT
}

1. Introduction. If $\mathfrak{S}$ is a Hilbert space the domain of an operator $T$ in $\mathfrak{S}$ will be denoted by $\mathfrak{D}(T)$. Let $S$ be a closed symmetric operator in $\mathfrak{S}$, that is, $\mathfrak{D}(S)$ is dense in $\mathfrak{S},(S f, g)=(f, S g)$ for all $f, g \in \mathfrak{D}(S)$, and the graph of $S$ is closed in $\mathfrak{S} \times \mathfrak{S}$. Suppose $S_{1}$ is a self-adjoint extension of $S$ in a (possibly larger) Hilbert space $\mathfrak{S}_{1}$. By this we mean $\mathfrak{S}_{1}$ contains $\mathfrak{S}_{\mathfrak{L}}$ as a subspace, and $S_{1}$ is self-adjoint in $\mathfrak{S}_{1}$ satisfying $S_{1} \supset S$. If $P_{1}$ is the orthogonal projection of $\mathfrak{W}_{1}$ onto $\mathfrak{S}$, the mapping $R$ from the nonreal complex numbers (which we denote hereafter by $\pi$ ) to the bounded operators $B$ in $\mathfrak{S}$ defined by

$$
R(l) h=P_{1}\left(S_{1}-l I\right)^{-1} h, \quad(h \in \mathfrak{S}, l \in \pi),
$$

is called a generalized resolvent of $S$. Here $I$ is the identity operator.

If $S_{1}$ is a self-adjoint extension of $S$ in $\mathfrak{S}_{1}$ and $E_{1}$ is its resolution of the identity,

$$
S_{1}=\int_{-\infty}^{\infty} \lambda d E_{1}(\lambda)
$$

then the operator-valued function $E$ defined on $\mathfrak{S}$ by

$$
E(\lambda) h=P_{1} E_{1}(\lambda) h, \quad(h \in \mathfrak{S},-\infty<\lambda<\infty),
$$

has the properties

$$
\begin{aligned}
E\left(\lambda_{1}\right) & \leqq E\left(\lambda_{2}\right) \quad \text { if } \lambda_{1}<\lambda_{2}, \\
E(\lambda+0) & =E(\lambda), \\
E(-\infty) & =0, \quad E(\infty)=I .
\end{aligned}
$$

(We assume $E_{1}$ is normalized so that $E_{1}(\lambda+0)=E_{1}(\lambda)$.) From the spectral theorem for $S_{1}$ it follows that

$$
\begin{aligned}
(S f, h) & =\int_{-\infty}^{\infty} \lambda d(E(\lambda) f, h), & (f \in \mathfrak{D}(S), h \in \mathfrak{S}), \\
\|S f\|^{2} & =\int_{-\infty}^{\infty} \lambda^{2} d(E(\lambda) f, f), & (f \in \mathfrak{D}(S)) .
\end{aligned}
$$

Received by the editors October 13, 1958.

(1) This work was supported in part by the National Science Foundation and the Office of Naval Research. 
A function $E$ from the real line to $B$ satisfying (a)-(c) above is called a generalized resolution of the identity. A spectral function of a closed symmetric operator $S$ in $\mathfrak{S}$ is a generalized resolution of the identity $E$ satisfying (d), (e) above. Naimark (See Appendix I of [1]) has shown that if $E$ is a spectral function of $S$ there exists a self-adjoint extension $S_{1}$ of $S$ in a Hilbert space $\mathfrak{S}_{1} \supset \mathfrak{S}$ with a resolution of the identity $E_{1}$ such that $E(\lambda)=P_{1} E_{1}(\lambda)$ on $\mathfrak{S}$. It is easy to see that a function $R$ from $\pi$ to $B$ is a generalized resolvent of a closed symmetric operator $S$ in $\mathfrak{E}$ if and only if it can be represented in the form

$$
R(l)=\int_{-\infty}^{\infty} \frac{d E(\lambda)}{\lambda-l}
$$

where $E$ is a spectral function of $S$.

In $\$ 2$ we consider the set $R$ of all generalized resolvents of a fixed closed symmetric operator $S$ in a Hilbert space $\mathfrak{S}$. Using the topology of weak operator convergence uniformly on compact subsets of $\pi$ we show that $R$ is a convex compact subset of the set of all analytic (in the weak operator sense) functions from $\pi$ to $B$. An application of the Krein-Milman theorem then shows that $R$ is the closed convex hull of its extreme points. One of us (Gilbert) has shown that if $S$ has finite and equal deficiency indices then the extreme points of $R$ are actually dense in $R$ in the topology of uniform operator convergence uniformly on compact subsets of $\pi$. The proof of this result will appear in a later paper.

Our principal interest in this paper is in generalized resolvents of an ordinary symmetric differential operator. Let $L$ be the formal operator

$$
L=p_{0} D^{n}+p_{1} D^{n-1}+\cdots+p_{n},
$$

where $D=d / d x$, the $p_{k}$ are complex-valued functions of class $C^{n-k}$ on an open real interval $a<x<b$ (the interval may be unbounded), and $p_{0}(x) \neq 0$ on $(a, b)$. We assume $L$ is formally self-adjoint. In $\mathfrak{S}=\mathfrak{Q}^{2}(a, b)$ let $T_{0}$ be the closure of the symmetric operator whose domain is the set of all functions of class $C^{\infty}$ on $(a, b)$ which vanish outside compact subsets of $(a, b)$, and whose value at each such function $u$ is $L u$. We shall call $T_{0}$ the minimal operator associated with $L$. It was shown by Coddington $[4 ; 5]$ that every generalized resolvent $R$ of $T_{0}$ is an integral operator of Carleman type

$$
R(l) f(x)=\int_{a}^{b} K(x, y, l) f(y) d y .
$$

The kernel $K$ of $R(l)$ can be decomposed into two parts $K=K_{0}+K_{1}$, where $K_{0}$ is a fixed fundamental solution for $L-l$, and $K_{1}$ is representable as $\left({ }^{2}\right)$

$$
K_{1}(x, y, l)=\sum_{j, k=1}^{n} \Psi_{j k}(l) s_{k}(x, l)\left[s_{j}(y, \bar{l})\right]^{-} .
$$

(2) [ ]- denotes the complex conjugate of [ ]. 
Here $s_{1}, \cdots, s_{n}$ form a basis for the solutions of $(L-l) u=0$ and satisfy the initial conditions $s_{j}^{(k-1)}(c, l)=\delta_{j k}$ for some fixed $c, a<c<b$. The matrix $\Psi$ is analytic in $\pi$ and satisfies $\Psi^{*}(l)=\Psi(\bar{l}), \operatorname{Im} \Psi(l) / \operatorname{Im} l \geqq 0$, where $\operatorname{Im} \Psi$ $=\left(\Psi-\Psi^{*}\right) / 2 i$. The matrix $\rho$ defined by

$$
\rho(\lambda)=\lim _{\epsilon \rightarrow+0} \frac{1}{\pi} \int_{0}^{\lambda} \operatorname{Im} \Psi(\nu+i \epsilon) d \nu
$$

exists, is nondecreasing (that is $\rho(\lambda) \geqq \rho(\mu)$ if $\lambda>\mu$ ), and is of bounded variation on any finite interval. The spectral function $E$ associated with $R$ via (1.1) is given by

$$
E(\Delta) f(x)=\int_{\Delta} \sum_{j, k=1}^{n} s_{k}(x, \lambda) \hat{f}_{j}(\lambda) d \rho_{j k}(\lambda),
$$

where here $\Delta=(\mu, \nu]$ is a finite interval, $E(\Delta)=E(\nu)-E(\mu), f \in \mathfrak{S}$ and vanishes outside a compact subset of $(a, b)$, and

$$
\hat{f}_{j}(\lambda)=\left(f, s_{j}(\lambda)\right)=\int_{a}^{b} f(x)\left[s_{j}(x, \lambda)\right]-d x .
$$

The matrix $\rho$ is called the spectral matrix associated with $E$ and $R$. Using the inner product

$$
(\zeta, \eta)=\int_{-\infty}^{\infty} \sum_{j, k=1}^{n} \zeta_{j}(\lambda)\left[\eta_{k}(\lambda)\right]-d \rho_{j k}(\lambda)
$$

for vector functions $\zeta=\left(\zeta_{1}, \cdots, \zeta_{n}\right), \eta=\left(\eta_{1}, \cdots, \eta_{n}\right)$ on the real line we can form the Hilbert space $\mathfrak{R}^{2}(\rho)$ consisting of all those $\zeta$ for which $\|\zeta\|=(\zeta, \zeta)^{1 / 2}$ $<\infty$. If $f \in \mathfrak{S}=\mathfrak{R}^{2}(a, b)$ and $\hat{f}=\left(\hat{f}_{1}, \cdots, \hat{f}_{n}\right)$ the mapping $f \rightarrow \hat{f}$ is an isometry of $\mathfrak{R}^{2}(a, b)$ into $\mathfrak{R}^{2}(\rho)$ whose inverse is given by

$$
f(x)=\int_{-\infty}^{\infty} \sum_{j, k=1}^{n} s_{k}(x, \lambda) \hat{j}_{j}(\lambda) d \rho_{j k}(\lambda) .
$$

For the proof of all these facts see [5].

In $\S 3$ we show that the mappings $R \rightarrow \Psi \rightarrow \rho$ are one-to-one and that convexity is preserved. Let the set of all $\Psi$ 's corresponding to all $R \in R$ be denoted by $\mathscr{M}$, and the set of all spectral matrices $\rho$ by $S$. The topology in $R$ goes over into uniform convergence on compact subsets for $\mathfrak{T}$, and into pointwise convergence at continuity points of the limit for $\mathcal{S}$. Thus, with the appropriate topology, $\mathfrak{T}$ and $\delta$ are the closed convex hulls of their extreme points.

A self-adjoint extension $S_{1}$ in $\mathfrak{S}_{1}$ of a closed symmetric operator $S$ in $\mathfrak{S}$ is said to be minimal if its resolution of the identity $E_{1}$ is such that the set of elements of the form $E_{1}(\lambda) f, f \in \mathfrak{S}_{2},-\infty<\lambda<\infty$, is fundamental in $\mathfrak{S}_{1}$, that is, the smallest subspace containing these elements is $\mathfrak{S}_{1}$. Naimark [8, 
Theorem 8] has shown that all minimal self-adjoint extensions of $S$ corresponding to a given spectral function $E$ of $S$ are unitarily equivalent. In $\$ 4$ we show that if $T_{0}$ is a closed symmetric ordinary differential operator and $E$ is a spectral function for $T_{0}$, a concrete realization of the minimal selfadjoint extension $S_{1}$ is given by the operator of multiplication by $\lambda$ in $\mathbb{R}^{2}(\rho)$, where $\rho$ is the spectral matrix for $E$. An application of this result shows that the isometry $f \rightarrow \hat{f}$ of $\mathbb{R}^{2}(a, b)$ into $\mathfrak{I}^{2}(\rho)$ is actually onto (i.e. a unitary mapping) if and only if $E$ is the resolution of the identity for a self-adjoint extension of $T_{0}$ in $\mathfrak{S}$ itself. This is the so-called inverse transform theorem, a proof of which was recently given by Levinson [7].

In $\$ 5$ we consider the case when the differential operator $L$ has continuous coefficients on a closed bounded interval $[a, b]$, and give a more detailed description of the matrices $\Psi$ which determine the generalized resolvents of $T_{0}$. They have the form

$$
\Psi=[C+F D]^{-1}[A+F B],
$$

$(\operatorname{Im} l>0)$,

where $A, B, C, D$ are matrices of entire functions uniquely determined by $L$ (and not depending on the particular spectral function $E$ ), and $F$ is an $n$ by $n$ matrix of analytic functions on $\operatorname{Im} l>0$ satisfying $\|F(l)\| \leqq 1$ (the operator norm is used). The set $\mathfrak{T}$ of all $\Psi$ is in a one-to-one correspondence with the set of all such matrices $F$. We prove that all minimal self-adjoint extensions of $T_{0}$, corresponding to $F$ which are continuous for $\operatorname{Im} l \geqq 0$ and which satisfy

$$
\sup _{\operatorname{Im} \rightarrow 0}\|F(l)\|<1,
$$

are unitarily equivalent. Indeed they are all unitarily equivalent to the direct sum $i D \oplus \cdots \oplus i D\left(n\right.$ times) on the space $\mathfrak{R}^{2}(-\infty, \infty) \oplus \cdots \oplus \mathbb{R}^{2}(-\infty, \infty)$ ( $n$ times).

We indicate in $\$ 6$ how spectral matrices of a $T_{0}$ defined on an open interval may be obtained as limits of spectral matrices for differential operators defined on closed bounded subintervals.

2. Generalized resolvents of a symmetric operator. Let $a$ be the set of all functions from the nonreal complex numbers $\pi$ to the bounded operators $B$ in a Hilbert space $\mathfrak{S}$, which are analytic in the weak operator topology (and hence analytic in the strong and uniform topologies). We give $a$ the topology of weak operator convergence uniformly on compact subsets of $\pi$. A subbase for the neighborhoods of an $A_{0} \in Q$ is the family of sets of the form

$$
\left\{A \in a:\left|\left(\left[A(l)-A_{0}(l)\right] f, g\right)\right|<\epsilon, l \in C\right\},
$$

where $f, g \in \mathfrak{S}, \epsilon>0$, and $C$ is a compact subset of $\pi$. A directed set $\left\{A_{\alpha}\right\}$, $A_{\alpha} \in Q$, will converge to an element $A \in Q$ in this topology if and only if $\left(A_{\alpha}(l) f, g\right)$ converges to $(A(l) f, g)$ uniformly on each compact subset of $\pi$, for each $f, g \in \mathfrak{L}$. The space $Q$ with this topology is easily seen to be a locally convex linear Hausdorff space. 
Let $S$ be a fixed closed symmetric operator in this Hilbert space $\mathfrak{E}$, and let $R$ denote the set of all its generalized resolvents.

TheOREM 1. $Q$ is a convex compact subset of $a$.

Proof. Equation (1.1) establishes a one-to-one correspondence between $R$ and the set of all spectral functions of $S$. The latter set is convex, as can be readily checked from the defining properties (a)-(e), in $\S 1$, and this implies via (1.1) that $R$ is convex.

Let $B_{l}$ denote the set of all bounded operators $B$ on $\mathfrak{F}$ satisfying $\|B\|$ $\leqq 1 /|\operatorname{Im} l|$. This set, for each fixed $l \in \pi$, is compact in the weak operator topology [6, p. 53], and therefore by the Tychonoff theorem the Cartesian product

$$
\prod_{l \in \pi} B_{l}
$$

is compact in the product topology. This product is the set of all functions $A$ from $\pi$ to $B$ satisfying $\|A(l)\| \leqq 1 /|\operatorname{Im} l|$, and the topology is that of weak operator convergence pointwise on $\pi$. It is clear from (1.1) that $R \subset \prod B_{l}$.

We show that $R$ is closed in $\prod Q_{l}$, and hence is compact in this topology. For the proof of this we use a characterization of generalized resolvents given by A. V. Straus [10]. His result is that a function $R$ from $\pi$ to $B$ such that $\mathfrak{D}(R(l))=\mathfrak{W}$ is a generalized resolvent of $S$ if and only if for every $l, \operatorname{Im} l>0$,

(i) $R(l) \psi$ is an analytic vector function for every $\psi \in \mathfrak{S} \theta$ range $(S-l I)$,

(ii) $\left(S^{*}-l I\right) R(l)=I$,

(iii) $\left\|\left(S^{*}-\bar{l} I\right) R(l)\right\| \leqq 1$,

(iv) $R^{*}(l)=R(\bar{l})$.

Let $\left\{R_{\alpha}\right\}, R_{\alpha} \in \mathcal{R}$, be a directed set which converges to an $R \in \Pi B_{l}$ weakly pointwise on $\pi$. All $R_{\alpha}$ are analytic on $\pi$, as can be seen from (1.1). Thus for each pair $f, g \in \mathfrak{S}$, the directed set of analytic functions $r_{\alpha}(l)=\left(R_{\alpha}(l) f, g\right)$ converges to $r(l)=(R(l) f, g)$ pointwise on $\pi$. If $C$ is any compact set in $\pi$, and $l \in C$,

$$
\left|r_{\alpha}(l)\right| \leqq\|f\|\|g\| /|\operatorname{Im} l| \leqq k(C)\|f\|\|g\|,
$$

where $k(C)$ is a constant depending only on $C$. This implies that the set of functions $\left\{r_{\alpha}\right\}$ is equicontinuous on any compact subset of the upper or lower half-plane. We infer from this, and the pointwise convergence of the $r_{\alpha}$, that $r_{\alpha} \rightarrow r$ uniformly on compact subsets of $\pi$. Thus $r$, and hence $R$, are analytic on $\pi$. In particular condition (i) is satisfied by $R$. Moreover on $R$ the topology of pointwise convergence is the same as the topology of uniform convergence on compact subsets of $\pi$. Hence the compactness of $R$ in $a$ follows from the compactness of $Q$ in $\Pi \otimes_{l}$.

To complete the proof we verify (ii)-(iv) for an $R$ which is a limit of a directed set $\left\{R_{\alpha}\right\}, R_{\alpha} \in R$, in $\prod \otimes_{l}$. Let $f \in \mathfrak{D}(S)$ and $h \in \mathfrak{S}$. Then 


$$
\begin{aligned}
(h, f) & =\left(\left(S^{*}-l I\right) R_{\alpha}(l) h, f\right)=\left(R_{\alpha}(l) h,(S-\bar{l} I) f\right) \\
& \rightarrow(R(l) h,(S-\bar{l} I) f),
\end{aligned}
$$

which implies $R(l) h \in \mathfrak{D}\left(S^{*}-l I\right)$, and $\left(S^{*}-l I\right) R(l)=I$, proving (ii). From (ii) we have

$$
\left(S^{*}-\bar{l} I\right) R(l)=I+(l-\bar{l}) R(l),
$$

and similarly for $R_{\alpha}(l)$. Thus if $g, h \in \mathfrak{S}$,

$$
\begin{aligned}
\left|\left(\left(S^{*}-\bar{l} I\right) R(l) h, g\right)\right| \leqq & \left|\left(\left(S^{*}-\bar{l} I\right) R_{\alpha}(l) h, g\right)\right| \\
& +|l-\bar{l}|\left|\left(\left[R(l)-R_{\alpha}(l)\right] h, g\right)\right| \\
\leqq & \|h\|\|g\|+\epsilon,
\end{aligned}
$$

where $\epsilon$ can be made arbitrarily small by a choice of $\alpha$. This proves (iii). Finally, if $g, h \in \mathfrak{S}$,

$$
\begin{aligned}
(R(l) h, g) & =\lim \left(R_{\alpha}(l) h, g\right)=\lim \left(h, R_{\alpha}^{*}(l) g\right) \\
& =\lim \left(h, R_{\alpha}(\bar{l}) g\right)=(h, R(\bar{l}) g),
\end{aligned}
$$

proving (iv), and Theorem 1.

We remark that if $\mathfrak{S}$ is separable the topology of $\mathscr{R}$ as a subset of $a$ is first countable. Indeed, if $\mathfrak{S}_{0}$ is a countable dense subset of $\mathfrak{S}$, and $I_{n}$, $I_{n} \subset I_{n+1}, n=1,2, \cdots$, is an exhaustion of $\pi$ consisting of pairs of rectangles in the upper and lower half-planes, the neighborhoods of an $R_{0}$ of the form

$$
\left\{R \in R:\left|\left(\left[R(l)-R_{0}(l)\right] \tilde{f}, \tilde{g}\right)\right|<1 / n, l \in I_{n}\right\}
$$

where $n=1,2, \cdots$, and $\tilde{f}, \tilde{g} \in \mathfrak{S}_{0}$, form a countable subbase for the neighborhoods of $R_{0}$.

An $R \in R$ is said to be an extreme point of $R$ if $R$ cannot be written as $R=c_{1} R_{1}+c_{2} R_{2}$ with $R_{1}, R_{2} \in R, R_{1} \neq R_{2}, c_{1}>0, c_{2}>0, c_{1}+c_{2}=1$. The KreinMilman theorem [2, p. 84] applied to the set $\mathcal{R}$ in $Q$ gives the following result.

THEOREM 2. $R$ is the closed convex hull of its extreme points.

Let a self-adjoint extension $S_{1}$ in $\mathfrak{S}_{1}$ of $S$ be called finite-dimensional if $\operatorname{dim}\left(\mathfrak{S}_{1} \ominus \mathfrak{S}\right)<\infty$. Naimark [9] has shown that all generalized resolvents of $S$ corresponding to finite-dimensional self-adjoint extensions of $S$ are extreme points of $\mathbb{R}$. In particular, if $S$ has self-adjoint extensions in $\mathfrak{S}$ itself, their resolvents will be extreme points of $R$. Gilbert has proved the following result, which will be the subject of a later paper.

Theorem 3. Suppose $S$ has finite and equal deficiency indices. Then given any $R \in R$ there exists a sequence $\left\{R_{n}\right\}$ of generalized resolvents of $S$, corresponding to finite-dimensional self-adjoint extensions of $S$, such that

$$
\left\|R_{n}(l)-R(l)\right\| \rightarrow 0, \quad(n \rightarrow \infty),
$$


uniformly on compact subsets of $\pi$.

3. Generalized resolvents of ordinary differential operators. We now consider a formally symmetric ordinary differential operator $L$ of order $n$ on an open interval $(a, b)$, and the minimal operator $T_{0}$ in $\mathfrak{E}=\mathbb{R}^{2}(a, b)$ associated with $L$. As mentioned in the introductory $\$ 1$ every generalized resolvent $R$ of $T_{0}$ is an integral operator of Carleman type with a kernel $K$ which can be decomposed into two parts $K=K_{0}+K_{1}$, where $K_{0}$ is a fixed fundamental solution for $L-l$ and $K_{1}$ is represented as

$$
K_{1}(x, y, l)=\sum_{j, k=1}^{n} \Psi_{j k}(l) s_{k}(x, l)\left[s_{j}(y, \bar{l})\right]^{-} .
$$

The $s_{j}$ satisfy $(L-l) s_{j}(x, l)=0$ and $s_{j}^{(k-1)}(c, l)=\delta_{j k}$ for some fixed $c, a<c<b$. It is intuitively clear that the behavior of a generalized resolvent $R$ of $T_{0}$, and the corresponding spectral matrix $\rho$ defined by

$$
\rho(\lambda)=\lim _{\epsilon \rightarrow+0} \frac{1}{\pi} \int_{0}^{\lambda} \operatorname{Im} \Psi(\nu+i \epsilon) d \nu,
$$

are completely determined by the matrix $\Psi=\left(\Psi_{j k}\right)$. It is the aim of this section to carry out the details of the correspondences $R \rightarrow \Psi \rightarrow \rho$. Let the set of all $\Psi$ 's corresponding via (3.1) to all $R \in R$ (the set of all generalized resolvents of $T_{0}$ ) be denoted by $\mathfrak{M}$, and the set of all spectral matrices defined via (3.2) by $\boldsymbol{S}$.

THEOREM 4. The correspondences $R \rightarrow \Psi \rightarrow \rho$ of $R \rightarrow \mathfrak{T} \rightarrow \mathcal{S}$ are all one-to-one. Both TI and S are convex.

Proof. Suppose there were two generalized resolvents $R_{1}, R_{2} \in R$ which have the same $\Psi \in \Re$. Then both $R_{1}, R_{2}$ would be integral operators with the same kernel, and therefore $R_{1}=R_{2}$. This shows that the map $R \rightarrow \Psi$ is one-toone.

It is clear from (3.2) that each $\Psi \in \mathscr{M}$ gives rise to a unique $\rho \in \mathcal{S}$. For each $\rho \in \mathcal{S}$ the spectral function $E$ of $T_{0}$ corresponding to $R$ is such that

$$
[E(\nu)-E(\mu)] f(x)=\int_{\mu}^{\nu} \sum_{j, k=1}^{n} s_{k}(x, \lambda) \hat{f}_{j}(\lambda) d \rho_{j k}(\lambda),
$$

for any $f \in \mathfrak{S}$ vanishing outside a compact subset of $(a, b)$. Here $\nu, \mu$ are continuity points of $E$, and $\hat{f}_{j}(\lambda)=\left(f, s_{j}(\lambda)\right)$. Suppose $\Psi_{1}, \Psi_{2} \in \mathscr{T}$ correspond via (3.2) to the same $\rho$. Since the correspondence $R \rightarrow E$ is one-to-one (see (1.1)) it would follow from (3.3) that $E_{1}(\nu)-E_{1}(\mu)=E_{2}(\nu)-E_{2}(\mu)$ at all continuity points of $E_{1}$ and $E_{2}$. Since the set of all discontinuity points of $E_{1}$ and $E_{2}$ is denumerable, it follows that there is a sequence $\left\{\mu_{n}\right\}$ of continuity points of $E_{1}$ and $E_{2}$ such that $\mu_{n} \rightarrow-\infty$. Then $E_{1}\left(\mu_{n}\right) \rightarrow 0, E_{2}\left(\mu_{n}\right) \rightarrow 0$, and we have $E_{1}(\nu)=E_{2}(\nu)$ at all continuity points of $E_{1}, E_{2}$. Since $E_{1}, E_{2}$ are continuous 
from the right $E_{1}=E_{2}$. This implies $R_{1}=R_{2}$, and therefore the map $\Psi \rightarrow \rho$ is one-to-one.

The definition of $\Psi$ via (3.1) and $\rho$ via (3.2) shows that if $c_{1} \geqq 0, c_{2} \geqq 0$, $c_{1}+c_{2}=1$, then $c_{1} R_{1}+c_{2} R_{2}$ corresponds to $c_{1} \Psi_{1}+c_{2} \Psi_{2}$ and $c_{1} \rho_{1}+c_{2} \rho_{2}$. Since $R$ is convex so are $\mathfrak{T}$ and $S$.

We now describe the topology on $\mathscr{T}$ and $\mathcal{S}$ which corresponds to the topology on $R$ as a subset of $Q$. We have shown that $R$ is a compact closed subset of $a$. Since $\mathfrak{S}=\mathbb{R}^{2}(a, b)$ is separable, the topology of $R$ is first countable, and is therefore determined completely by the convergent sequences in $R$.

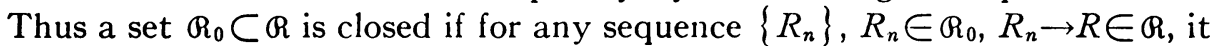
follows that $R \in R_{0}$.

Theorem 5. Let $R_{n}, R \in R$ correspond to $\Psi_{n}, \Psi \in \mathscr{T}, n=1,2, \cdots$. The following are equivalent:

(a) $R_{n} \rightarrow R$ weakly, uniformly on compact subsets of $\pi$,

(b) $R_{n} \rightarrow R$ uniformly, uniformly on compact subsets of $\pi$,

(c) $\Psi_{n} \rightarrow \Psi$ uniformly on compact subsets of $\pi$.

Note: The norm $\|\Psi\|$ of a matrix $\Psi=\left(\Psi_{j k}\right)$ is defined by

$$
\|\Psi\|=\sum_{j, k=1}^{n}\left|\Psi_{j k}\right| \text {. }
$$

By $\Psi_{n} \rightarrow \Psi$ we mean $\left\|\Psi_{n}-\Psi\right\| \rightarrow 0$.

Proof of Theorem 5. In order to prove these equivalences it is sufficient, by compactness, to prove these results for a disk about each point $l_{0} \in \pi$. Also since $R^{*}(l)=R(\bar{l}), \Psi^{*}(l)=\Psi(\bar{l})$, it follows that it is sufficient to consider only $l_{0}$ in the upper half-plane. Let $l_{0}$ be such a point.

In proving $R$ is an integral operator of Carleman type [5] it was shown that $R$ could be represented as

$$
R(l) f=G(l) f+\sum_{j=1}^{\omega^{-}} \sum_{k=1}^{\omega^{+}} \Phi_{j k}(l)\left(f, \psi_{j}(\bar{l})\right) \phi_{k}(l) .
$$

The operator $G(l)$ is an integral operator which is a right inverse of $T_{0}^{*}-l$, analytic in $\pi$, and satisfies $\|G(l)\| \leqq 1 /|\operatorname{Im} l|$. The functions $\phi_{k}, \psi_{j}$ are defined by

$$
\begin{array}{ll}
\phi_{k}(l)=\left[I+\left(l-l_{0}\right) G(l)\right] \phi_{k}\left(l_{0}\right), & \left(k=1, \cdots, \omega^{+}\right), \\
\psi_{j}(\bar{l})=\left[I+\left(\bar{l}-\bar{l}_{0}\right) G(\bar{l})\right] \psi_{j}\left(\bar{l}_{0}\right), & \left(j=1, \cdots, \omega^{-}\right),
\end{array}
$$

and are analytic bases for the eigenspaces

$$
\begin{aligned}
& \mathfrak{l}(l)=\left\{u \in \mathfrak{D}\left(T_{0}^{*}\right) \mid T_{0}^{*} u=l u\right\}, \\
& \mathfrak{l}(\bar{l})=\left\{u \in \mathfrak{D}\left(T_{0}^{*}\right) \mid T_{0}^{*} u=\bar{l} u\right\},
\end{aligned}
$$

respectively for $\left|l-l_{0}\right|<\operatorname{Im} l_{0} / 2$. The sets $\left\{\phi_{k}\left(l_{0}\right)\right\}$ and $\left\{\psi_{j}\left(\bar{l}_{0}\right)\right\}$ can be 
chosen to be orthonormal bases for $\&\left(l_{0}\right)$ and $\&\left(\bar{l}_{0}\right)$ respectively. The matrix $\Phi=\left(\Phi_{j k}\right)$ is analytic for $\left|l-l_{0}\right|<\operatorname{Im} l_{0} / 2$. We note that from (3.5) we have

$$
\left\|\phi_{k}(l)-\phi_{k}\left(l_{0}\right)\right\| \leqq \frac{\left|l-l_{0}\right|}{|\operatorname{Im} l|}\left\|\phi_{k}\left(l_{0}\right)\right\|<\frac{2\left|l-l_{0}\right|}{\operatorname{Im} l_{0}}<1,
$$

if $\left|l-l_{0}\right|<\operatorname{Im} l_{0} / 2$. In particular $\left\|\phi_{k}(l)\right\|<2$. Similar estimates hold for $\psi_{j}(\bar{l})$.

From (3.4) we see that if $\Phi_{n}=\left(\Phi_{j k}^{n}\right)$ corresponds to $R_{n}$ then

$$
\begin{aligned}
\left(R_{n}(l) \psi_{p}\left(\bar{l}_{0}\right), \phi_{q}\left(l_{0}\right)\right)- & \left(R(l) \psi_{p}\left(\bar{l}_{0}\right), \phi_{q}\left(l_{0}\right)\right) \\
& =\sum_{j=1}^{\omega^{-}} \sum_{k=1}^{\omega^{+}}\left[\Phi_{j k}^{n}(l)-\Phi_{j k}(l)\right]\left(\psi_{p}\left(\bar{l}_{0}\right), \psi_{j}(\bar{l})\right)\left(\phi_{k}(l), \phi_{q}\left(l_{0}\right)\right) .
\end{aligned}
$$

Since the matrices with elements $\left(\psi_{p}\left(\bar{l}_{0}\right), \psi_{j}\left(\bar{l}_{0}\right)\right)$ and $\left(\phi_{k}\left(l_{0}\right), \phi_{q}\left(l_{0}\right)\right)$ are the identity matrices, and since the matrices with elements $\left(\psi_{p}\left(\bar{l}_{0}\right), \psi_{j}(\bar{l})\right)$ and $\left(\phi_{k}(l), \phi_{q}\left(l_{0}\right)\right)$ are continuous at $l_{0}$ by (3.6), it follows that there is a closed disk $\Delta_{0}$ about $l_{0}$ contained in $\left|l-l_{0}\right|<\operatorname{Im~} l_{0} / 2$ such that the latter matrices are invertible, with continuous inverses there. It then follows from (3.7) that if $R_{n} \rightarrow R$ weakly, uniformly on $\Delta_{0}$, then $\Phi_{n} \rightarrow \Phi$ uniformly on $\Delta_{0}$. Now suppose $\Phi_{n} \rightarrow \Phi$ uniformly on $\Delta_{0}$. Then from (3.4) it follows that

$$
\left\|R_{n}(l) f-R(l) f\right\| \leqq 4\|f\|\left\|\Phi_{n}(l)-\Phi(l)\right\|,
$$

which shows that $\left\|R_{n}(l)-R(l)\right\| \rightarrow 0$ uniformly on $\Delta_{0}$. This proves that (a) is equivalent to (b).

In order to prove the equivalence of (b) and (c) we explore the relationship between $\Phi$ and $\Psi$ in $\Delta_{0}$. From the representation (3.4) we see that the kernel $K$ of $R(l)$ can be written as

$$
K(x, y, l)=G(x, y, l)+\sum_{j=1}^{\omega^{-}} \sum_{k=1}^{\omega^{+}} \Phi_{j k}(l) \phi_{k}(x, l)\left[\psi_{j}(y, \bar{l})\right]-
$$

where $G$ is the kernel of $G(l)$. This kernel $G$ can be written as $G=K_{0}+G_{0}$, where $K_{0}$ is the fundamental solution of $L-l$ referred to in the decomposition of $K$, and where $G_{0}$ has the form

$$
G_{0}(x, y, l)=\sum_{j, k=1}^{n} \Phi_{j k}^{0}(l) s_{k}(x, l)\left[s_{j}(y, \bar{l})\right]^{-}
$$

see [5]. Sirice $s_{1}(l), \cdots, s_{n}(l)$ is a basis for the solutions of $(L-l) u=0$, and $(L-l) \phi_{k}(l)=0,(L-\bar{l}) \psi_{j}(\bar{l})=0$, we have

$$
\begin{aligned}
\phi_{k}(x, l) & =\sum_{q=1}^{n} M_{k q}(l) s_{q}(x, l), & \left(k=1, \cdots, \omega^{+}\right), \\
\psi_{j}(y, \bar{l}) & =\sum_{p=1}^{n} N_{j p}(\bar{l}) s_{p}(y, \bar{l}), & \left(j=1, \cdots, \omega^{-}\right),
\end{aligned}
$$


where the matrices $M(l)=\left(M_{q k}(l)\right)$ and $N(\bar{l})=\left(N_{j p}(\bar{l})\right)$ have ranks $\omega^{+}$and $\omega^{-}$ respectively. Since $s_{q}^{(j-1)}(c, l)=\delta_{j q}$ we see that

$$
M_{k j}(l)=\phi_{k}^{(j-1)}(c, l), \quad N_{j k}(\bar{l})=\psi_{j}^{(k-1)}(c, \bar{l}) .
$$

Hence $M(l)$ and $N^{*}(\bar{l})$ are analytic in $l$ for $\left|l-l_{0}\right|<\operatorname{Im} l_{0} / 2$. Placing (3.11) into (3.9) we obtain

$$
K(x, y, l)=K_{0}(x, y, l)+G_{0}(x, y, l)+\sum_{p, q=1}^{n} \Phi_{p q}^{+}(l) s_{q}(x, l)\left[s_{p}(y, \bar{l})\right]^{-}
$$

where if $\Phi_{+}(l)=\left(\Phi_{p q}^{+}(l)\right)$,

$$
\Phi_{+}(l)=N^{*}(\bar{l}) \Phi(l) M(l) .
$$

Since $K=K_{0}+K_{1}$, where $K_{1}$ is given by (3.1), we see that

$$
\Psi(l)=\Phi_{0}(l)+\Phi_{+}(l),
$$

where $\Phi_{0}(l)=\left(\Phi_{j k}^{0}(l)\right)$ is the matrix appearing in (3.10).

Now assume $R_{n} \rightarrow R$ uniformly on $\Delta_{0}$. Then $\Phi_{n} \rightarrow \Phi$ uniformly on $\Delta_{0}$ and from (3.12), (3.13) we see that

$$
\Psi_{n}(l)-\Psi(l)=\Phi_{+n}(l)-\Phi_{+}(l)=N^{*}(\bar{l})\left[\Phi_{n}(l)-\Phi(l)\right] M(l),
$$

which tends to zero uniformly on $\Delta_{0}$. Conversely, let $\Psi_{n}(l) \rightarrow \Psi(l)$ uniformly on $\Delta_{0}$. Since $M(l)$ has rank $\omega^{+}$and $N^{*}(\bar{l})$ rank $\omega^{-}, M(l)$ has a right inverse and $N^{*}(\bar{l})$ has a left inverse for each $l \in \Delta_{0}$. It then follows from (3.14) that $\Phi_{n}(l) \rightarrow \Phi(l)$ pointwise on $\Delta_{0}$, and this in turn implies, by virtue of (3.8), that $R_{n}(l) \rightarrow R(l)$ uniformly, pointwise on $\Delta_{0}$. In particular $R_{n}(l) \rightarrow R(l)$ weakly, pointwise on $\Delta_{0}$. But we have already seen in the proof of Theorem 1 that pointwise convergence implies uniform convergence on compact subsets of $\pi$. Thus $R_{n} \rightarrow R$ weakly, uniformly on $\Delta_{0}$, and from the equivalence of (a) and (b) we have $R_{n} \rightarrow R$ uniformly, uniformly on $\Delta_{0}$. This shows that (b) is equivalent to (c), and completes the proof of the theorem.

Let us use the topology on $\mathfrak{T}$ of convergence in the metric induced by the norm, uniformly on compact subsets of $\pi$. A consequence of Theorem 5 is that the mapping $R \rightarrow \Psi$ of $R$ onto $\mathbb{T}$ is a homeomorphism. The extreme points of $R$ map onto the extreme points of $\mathfrak{T}$. Thus the following result is a consequence of Theorem 2 .

THEOREM 6. $\mathfrak{T}$ is the closed convex hull of its extreme points.

We now investigate the correspondence $\Psi \in \mathscr{T} \rightarrow \rho \in \mathcal{S}$. Since any $\Psi \in \mathscr{M}$ is analytic on $\pi, \Psi^{*}(l)=\Psi(\bar{l}), \operatorname{Im} \Psi(l) / \operatorname{Im} l \geqq 0$, it may be represented in the form

$$
\Psi(l)=\alpha+l \beta+\int_{-\infty}^{\infty} \frac{\lambda l+1}{\lambda-l} d \sigma(\lambda)
$$

where $\alpha, \beta$ are constant Hermitian matrices, $\beta \geqq 0$, and $\sigma$ is a Hermitian 
matrix function of bounded variation which is nondecreasing in the sense that $\sigma(\lambda) \geqq \sigma(\mu)$ if $\lambda>\mu$. If $\sigma$ is normalized so that $\sigma(\lambda+0)=\sigma(\lambda), \sigma(0)=0$, this representation is unique; see [5]. We first prove a preliminary lemma concerning matrices $\Psi$ representable in the form (3.15).

LemMa. Let $\left\{\Psi_{n}\right\}$ be a sequence of matrices representable in the form (3.15), corresponding to $\left\{\alpha_{n}\right\},\left\{\beta_{n}\right\}$, and $\left\{\sigma_{n}\right\}$. Suppose $\Psi_{n} \rightarrow \Psi$ pointwise on $\pi$, and let $\Psi$ correspond via (3.15) to $\alpha, \beta, \sigma$. Then

(a) $\alpha_{n} \rightarrow \alpha$,

(b) $\beta_{n}+\int_{-\infty}^{\infty} d \sigma_{n}(\lambda) \rightarrow \beta+\int_{-\infty}^{\infty} d \sigma(\lambda)$,

(c) $0 \leqq \int_{-\infty}^{\infty} d \sigma_{n}(\lambda) \leqq k I$, for some constant $k, 0<k<\infty$,

(d) $\sigma_{n}(\lambda)-\sigma_{n}(\mu) \rightarrow \sigma(\lambda)-\sigma(\mu)$, at continuity points $\lambda$, $\mu$ of $\sigma$.

Note. In (c) $I$ is the identity matrix. This (c) is equivalent to

$$
\int_{-\infty}^{\infty}\left\|d \sigma_{n}(\lambda)\right\| \leqq k^{\prime}, \quad \text { for some constant } k^{\prime} .
$$

Proof of the lemma. We have

$$
\Psi_{n}(i)=\alpha_{n}+i \beta_{n}+i \int_{-\infty}^{\infty} d \sigma_{n}(\lambda)
$$

and therefore

$$
\alpha_{n}=\operatorname{Re} \Psi_{n}(i) \rightarrow \operatorname{Re} \Psi(i)=\alpha,
$$

and

$$
\beta_{n}+\int_{-\infty}^{\infty} d \sigma_{n}(\lambda)=\operatorname{Im} \Psi_{n}(i) \rightarrow \operatorname{Im} \Psi(i)=\beta+\int_{-\infty}^{\infty} d \sigma(\lambda)
$$

proving (a) and (b). Since $\beta_{n} \geqq 0$ and $\int_{-\infty}^{\infty} d \sigma_{n}(\lambda) \geqq 0$ we see that (c) follows from (b).

From $\left(c^{\prime}\right)$, and a theorem due to Helly, there exists a subsequence $\left\{\sigma_{n_{k}}\right\}$ which converges to a nondecreasing matrix $\tilde{\sigma}$ point-wise on $(-\infty, \infty)$. Then

$$
\int_{-\infty}^{\infty} \frac{d \sigma_{n_{k}}(\lambda)}{\lambda-l} \rightarrow \int_{-\infty}^{\infty} \frac{d \tilde{\sigma}(\lambda)}{\lambda-l}
$$

for each $l \in \pi$. Indeed $|\lambda-l|^{-1} \rightarrow 0$ as $|\lambda| \rightarrow \infty$ and $\left(c^{\prime}\right)$ imply that

$$
\int_{|\lambda|>\Lambda} \frac{d \sigma_{n_{k}}(\lambda)}{\lambda-l} \rightarrow 0, \quad(\Lambda \rightarrow \infty),
$$

uniformly in $\left\{n_{k}\right\}$. Since $\int_{-\infty}^{\infty}\|d \tilde{\sigma}(\lambda)\| \leqq k^{\prime}$ from $\left(\mathrm{c}^{\prime}\right)$ it follows that the integral on the right side of (3.16) exists. From the Helly integration theorem we have

$$
\int_{|\lambda| \leq \Delta} \frac{d \sigma_{n_{k}}(\lambda)}{\lambda-l} \rightarrow \int_{|\lambda| \leq \Delta} \frac{d \tilde{\sigma}(\lambda)}{\lambda-l} .
$$


Therefore given any $\epsilon>0$ there exists a $\Lambda>0$ such that

$$
\left\|\int_{|\lambda|>\Lambda} \frac{d \sigma_{n_{k}}(\lambda)}{\lambda-l}\right\|+\left\|\int_{|\lambda|>\Lambda} \frac{d \tilde{\sigma}(\lambda)}{\lambda-l}\right\|<\epsilon,
$$

and for such a $\Lambda$ there exists an $N>0$ such that

$$
\left\|\int_{|\lambda| \leq \Lambda} \frac{d \sigma_{n_{k}}(\lambda)}{\lambda-l}-\int_{|\lambda| \leq \Lambda} \frac{d \tilde{\sigma}(\lambda)}{\lambda-l}\right\|<\epsilon, \quad n_{k}>N .
$$

Combining (3.17) and (3.18) we obtain (3.16).

Now

$$
\begin{aligned}
\Psi_{n_{k}}(l) & =\alpha_{n_{k}}+l \beta_{n_{k}}+\int_{-\infty}^{\infty} \frac{\lambda l+1}{\lambda-l} d \sigma_{n_{k}}(\lambda) \\
& =\alpha_{n_{k}}+l\left[\beta_{n_{k}}+\int_{-\infty}^{\infty} d \sigma_{n_{k}}(\lambda)\right]+\left(l^{2}+1\right) \int_{-\infty}^{\infty} \frac{d \sigma_{n_{k}}(\lambda)}{\lambda-l} \\
& \rightarrow \alpha+l\left[\beta+\int_{-\infty}^{\infty} d \sigma(\lambda)\right]+\left(l^{2}+1\right) \int_{-\infty}^{\infty} \frac{d \tilde{\sigma}(\lambda)}{\lambda-l}
\end{aligned}
$$

using (a), (b), and (3.16). However

$$
\Psi_{n_{k}}(l) \rightarrow \Psi(l)=\alpha+l\left[\beta+\int_{-\infty}^{\infty} d \sigma(\lambda)\right]+\left(l^{2}+1\right) \int_{-\infty}^{\infty} \frac{d \sigma(\lambda)}{\lambda-l},
$$

which implies that

$$
\int_{-\infty}^{\infty} \frac{d \tilde{\sigma}(\lambda)}{\lambda-l}=\int_{-\infty}^{\infty} \frac{d \sigma(\lambda)}{\lambda-l}
$$

for all $l \in \pi$, and from the Stieltjes inversion formula we see that

$$
\tilde{\sigma}(\lambda)-\tilde{\sigma}(\mu)=\sigma(\lambda)-\sigma(\mu)
$$

at continuity points of $\sigma$. Since every convergent subsequence of $\left\{\sigma_{n}(\lambda)-\sigma_{n}(\mu)\right\}$ tends to the same limit we obtain (d), and the lemma is proved.

Recall that the correspondence $\Psi \in \mathfrak{N} \rightarrow \rho \in S$ is one-to-one. The relationship between $\rho$ and the $\sigma$ of (3.15) is

$$
\rho(\lambda)-\rho(\mu)=\int_{\mu}^{\lambda}\left(1+\nu^{2}\right) d \sigma(\nu)
$$

at continuity points $\lambda, \mu$ of $\sigma$.

Theorem 7. Let $\Psi_{n}, \Psi \in \mathfrak{M}$ correspond to $\rho_{n}, \rho \in \mathcal{S}$ respectively. Then $\Psi_{n} \rightarrow \Psi$ uniformly on compact subsets of $\pi$ if and only if

$$
\rho_{n}(\lambda)-\rho_{n}(\mu) \rightarrow \rho(\lambda)-\rho(\mu)
$$


at continuity points $\lambda, \mu$ of $\rho$.

Proof. First suppose $\Psi_{n} \rightarrow \Psi$ uniformly on compact subsets of $\pi$. Using the representation (3.15) we see from the lemma that $\sigma_{n}(\lambda)-\sigma_{n}(\mu) \rightarrow \sigma(\lambda)-\sigma(\mu)$ at continuity points of $\sigma$. This implies (3.20). Indeed there exists a subsequence $\left\{\sigma_{n_{k}}\right\}$ converging to some limit $\tilde{\sigma}$, and using the Helly integration theorem we obtain

$$
\begin{aligned}
\rho_{n_{k}}(\lambda)-\rho_{n_{k}}(\mu) & =\int_{\mu}^{\lambda}\left(1+\nu^{2}\right) d \sigma_{n_{k}}(\nu) \\
& \rightarrow \int_{\mu}^{\lambda}\left(1+\nu^{2}\right) d \tilde{\sigma}(\nu)=\int_{\mu}^{\lambda}\left(1+\nu^{2}\right) d \sigma(\nu)=\rho(\lambda)-\rho(\mu),
\end{aligned}
$$

since $\tilde{\sigma}(\lambda)-\tilde{\sigma}(\mu)=\sigma(\lambda)-\sigma(\mu)$ at continuity points of $\sigma$. Thus every convergent subsequence of $\left\{\rho_{n}(\lambda)-\rho_{n}(\mu)\right\}$ converges to the same limit, proving (3.20).

Conversely suppose (3.20) is valid. Then since the map $\rho \rightarrow \Psi$ is one-to-one there exist unique $\alpha_{n}, \beta_{n}, \alpha, \beta$ such that $\Psi_{n}$ is represented via (3.15) by $\alpha_{n}, \beta_{n}$, $\sigma_{n}$, and $\Psi$ is represented by $\alpha, \beta, \sigma$. From (3.19) it is clear that

$$
\sigma(\lambda)-\sigma(\mu)=\int_{\mu}^{\lambda} \frac{d \rho(\nu)}{1+\nu^{2}}
$$

at continuity points, and by the reasoning given in the first part of the proof we see $\sigma_{n}(\lambda)-\sigma_{n}(\mu) \rightarrow \sigma(\lambda)-\sigma(\mu)$ at continuity points of $\sigma$. Since $\mathfrak{T C}$ is compact, and its topology is first countable, it is sequentially compact. Hence there is a subsequence $\left\{\Psi_{n_{k}}\right\}$ of $\left\{\Psi_{n}\right\}$ which converges uniformly on compact subsets of $\pi$ to a limit $\tilde{\Psi} \in \mathscr{T}$. If $\tilde{\sigma}$ corresponds to $\tilde{\Psi}$ via (3.15) we see from the lemma that $\sigma_{n_{k}}(\lambda)-\sigma_{n_{k}}(\mu) \rightarrow \tilde{\sigma}(\lambda)-\tilde{\sigma}(\mu)$, and hence $\tilde{\sigma}(\lambda)-\tilde{\sigma}(\mu)=\sigma(\lambda)-\sigma(\mu)$ at continuity points of $\sigma$, which implies $\sigma(\lambda)=\tilde{\sigma}(\lambda)$. Thus $\tilde{\rho}(\lambda)-\tilde{\rho}(\mu)=\rho(\lambda)-\rho(\mu)$ at continuity points of $\rho$, which in turn implies $\tilde{\Psi}=\Psi$ (see the argument in Theorem 4). The above shows that every convergent subsequence of $\left\{\Psi_{n}\right\}$ tends to the same limit $\Psi$, and therefore $\Psi_{n} \rightarrow \Psi$ pointwise on $\pi$. (No subsequence can tend to infinity at a point $l_{0} \in \pi$, for $\mathscr{T}$ is sequentially compact.) But this implies $\Psi_{n} \rightarrow \Psi$ uniformly on compact subsets of $\pi$, since $\left\{\Psi_{n}\right\}$ is a normal family. Indeed

$$
\Psi_{n}(l)=\alpha_{n}+l\left[\beta_{n}+\int_{-\infty}^{\infty} d \sigma_{n}(\lambda)\right]+\left(l^{2}+1\right) \int_{-\infty}^{\infty} \frac{d \sigma_{n}(\lambda)}{\lambda-l},
$$

and hence

$$
\left\|\Psi_{n}(l)\right\| \leqq\left\|\alpha_{n}\right\|+|l|\left\|\beta_{n}+\int_{-\infty}^{\infty} d_{\sigma_{n}}(\lambda)\right\|+\left|\frac{l^{2}+1}{\operatorname{Im} l}\right| \int_{-\infty}^{\infty}\left\|d \sigma_{n}(\lambda)\right\|,
$$

which is bounded on any compact set $C$ in $\pi$ by (a), (b), (c') of the lemma. 
This completes the proof of the theorem.

By defining a set $\delta_{0} \subset \delta$ to be closed if for every sequence $\left\{\rho_{n}\right\}$ in $\delta_{0}$ such that $\rho_{n}(\lambda)-\rho_{n}(\mu) \rightarrow \rho(\lambda)-\rho(\mu)$, at continuity points $\lambda, \mu$ of $\rho \in \mathcal{S}$, it follows $\rho \in \mathcal{S}_{0}$, we obtain a topology for $S$, and with this topology $S$ is homeomorphic to $\mathfrak{T}$. If $c_{1} \geqq 0, c_{2} \geqq 0, c_{1}+c_{2}=1$, then $c_{1} \Psi_{1}+c_{2} \Psi_{2} \in \mathfrak{N}$ corresponds to $c_{1} \rho_{1}$ $+c_{2} \rho_{2} \in S$. Thus Theorem 6 implies

THEOREM 8. $S$ is the closed convex hull of its extreme points.

4. Minimal self-adjoint extensions of ordinary differential operators. Let, as in $\S 3, T_{0}$ be the minimal operator in $\mathfrak{E}=\ell^{2}(a, b)$ associated with a formally self-adjoint ordinary differential operator $L$ on $(a, b)$. A self-adjoint extension $S_{1}$ of $T_{0}$ in a Hilbert space $\mathfrak{S}_{1} \supset \mathfrak{S}$ is said to be minimal if its resolution of the identity $E_{1}$ is such that the set $\left\{E_{1}(\lambda) f: f \in \mathfrak{S},-\infty<\lambda<\infty\right\}$ is fundamental in $\mathfrak{S}_{1}$. According to Naimark [8, Theorem 8] all minimal self-adjoint extensions of $T_{0}$, corresponding to a given spectral function $E$ of $T_{0}$, are unitarily equivalent. Indeed if $S_{1}, S_{2}$ are two such minimal self-adjoint extensions on $\mathfrak{S}_{1} \supset \mathfrak{S}_{2} \mathfrak{S}_{2} \supset \mathfrak{S}$ respectively, then there exists an isometry $U$ of $\mathfrak{S}_{1}$ onto $\mathfrak{S}_{2}$ leaving $\mathfrak{S}$ invariant, and such that $S_{2}=U S_{1} U^{-1}$.

Let $E$ be a spectral function for $T_{0}$, and let $\rho \in S$ correspond to $E$. Then the map $f \in \mathfrak{S} \rightarrow \hat{f} \in \mathfrak{R}^{2}(\rho)$ is an isometry $V$ of $\mathfrak{S}$ onto $V \mathfrak{S} \subset \mathbb{R}^{2}(\rho)$; see the introduction. Let $\hat{T}_{0}$ be the operator defined in $\mathfrak{R}^{2}(\rho)$ with domain $V \mathfrak{D}\left(T_{0}\right)$ by $\hat{T}_{0} V f(\lambda)=\lambda V f(\lambda)$, for each $f \in \mathfrak{D}\left(T_{0}\right)$. Then $\hat{T}_{0}=V T_{0} V^{-1}$. Indeed $\mathfrak{D}\left(V T_{0} V^{-1}\right)$ is the set of all $\zeta \in \mathfrak{R}^{2}(\rho)$ such that $V^{-1} \zeta \in \mathfrak{D}\left(T_{0}\right)$, i.e. $\zeta \in V \mathfrak{D}\left(T_{0}\right)$. If $\zeta \in V \mathfrak{D}\left(T_{0}\right)$ then $\zeta=\hat{f}$ for some $f \in \mathfrak{D}\left(T_{0}\right)$, and $V T_{0} V^{-1} \zeta=\left[T_{0} f\right]^{\wedge}\left({ }^{3}\right)$. Now, using condition (d) satisfied by $E$, and the explicit form of the $E(\lambda)$ (see the introduction), we have for any $g \in \mathfrak{S}$,

$$
\begin{aligned}
\left(\left[T_{0} f\right]^{\wedge}, \hat{g}\right) & =\left(T_{0} f, g\right)=\int_{-\infty}^{\infty} \lambda d(E(\lambda) f, g) \\
& =\int_{-\infty}^{\infty} \sum_{j, k=1}^{n} \lambda\left[\hat{g}_{k}(\lambda)\right]-\hat{f}_{j}(\lambda) d \rho_{j k}(\lambda) \\
& =\left(\hat{T}_{0} \hat{f}, \hat{g}\right) .
\end{aligned}
$$

Thus $\hat{T}_{0} \hat{f}=\left[T_{0} f\right]^{\wedge}+\eta$, where $\eta \in \mathfrak{R}^{2}(\rho) \ominus V \mathfrak{S}$. Using condition (e) we find

$$
\begin{aligned}
\left\|\left[T_{0} f\right]^{\wedge}\right\|^{2} & =\left\|T_{0} f\right\|^{2}=\int_{-\infty}^{\infty} \lambda^{2} d(E(\lambda) f, f) \\
& =\int_{-\infty}^{\infty} \sum_{j, k=1}^{n} \lambda^{2}\left[\hat{f}_{k}(\lambda)\right]-\hat{f}_{j}(\lambda) d \rho_{j k}(\lambda) \\
& =\left\|\hat{T}_{0} f\right\|^{2},
\end{aligned}
$$

(3) [ ]^ denotes $V[]$. 
and since $\left\|\hat{T}_{0} \hat{f}\right\|^{2}=\left\|\left[T_{0} f\right]^{\wedge}\right\|^{2}+\|\eta\|^{2}$ we see that $\eta=0$. Therefore $\left[T_{0} f\right]^{\wedge}$ $=\hat{T}_{0} \hat{f}$, or $V T_{0} f=\hat{T}_{0} V f$, or $\hat{T}_{0}=V T_{0} V^{-1}$. Thus $\hat{T}_{0}$ is a unitary copy of $T_{0}$ in the copy $V \mathfrak{S}$ of $\mathfrak{E}$.

Let $S_{1}$ be the operator in $\mathfrak{R}^{2}(\rho)$ with domain $\mathfrak{D}\left(S_{1}\right)$ the set of all $\zeta \in \mathfrak{R}^{2}(\rho)$ such that $\lambda \zeta \in \mathbb{R}^{2}(\rho)$, and which is defined by $S_{1} \zeta(\lambda)=\lambda \zeta(\lambda)$. This operator is self-adjoint in $\mathfrak{R}^{2}(\rho)$, and its resolution of the identity $E_{1}$ is such that if $\Delta=(\mu, \lambda]$ is a real interval, and $E_{1}(\Delta)=E_{1}(\lambda)-E_{1}(\mu)$, then $E_{1}(\Delta) \zeta=\chi_{\Delta} \zeta$, where $\chi_{\Delta}$ is the characteristic function of the interval $\Delta$. See, for example $[1$, pp. 205, 206].

Let $P_{1}$ be the orthogonal projection of $\mathfrak{R}^{2}(\rho)$ onto $V \mathfrak{S}$. Then $V E(\Delta) V^{-1} \hat{f}$ $=P_{1} E_{1}(\Delta) \hat{f}$ for all $\hat{f} \in V \mathfrak{S}$. Indeed $V E(\Delta) V^{-1} \hat{f}=[E(\Delta) f]^{\wedge}$, whereas $P_{1} E_{1}(\Delta) \hat{f}$ $=P_{1} \chi_{\Delta} \hat{f}$. If $f, g \in \mathfrak{S}$, are continuous and vanish outside compact subsets, then

$$
\begin{aligned}
\left([E(\Delta) f]^{\wedge}, \hat{g}\right) & =(E(\Delta) f, g)=\int_{\Delta} \sum_{j, k=1}^{n}\left[\hat{g}_{k}(\lambda)\right]-\hat{f}_{j}(\lambda) d \rho_{j k}(\hat{\lambda}) \\
& =\left(\chi_{\Delta} \hat{f}, \hat{g}\right) .
\end{aligned}
$$

Thus $[E(\Delta) f]^{\wedge}=\chi_{\Delta} \hat{f}+\eta$, where $\eta \in \mathbb{R}^{2}(\rho) \ominus V \mathfrak{W}$, and hence $[E(\Delta) f]^{\wedge}$ $=P_{1}[E(\Delta) f]^{\wedge}=P_{1} \chi_{\Delta} \hat{f}$, or $V E(\Delta) V^{-1}=P_{1} E_{1}(\Delta)$ on $V \mathfrak{S}$. As a consequence we see that $S_{1}$ is a self-adjoint extension of $V T_{0} V^{-1}$ corresponding to the spectral function $V E V^{-1}$.

THEOREM 9. $S_{1}$ is a minimal self-adjoint extension of $\hat{T}_{0}=V T_{0} V^{-1}$ corresponding to the spectral function $V E V^{-1}$ on $V \mathfrak{S}$.

Proof. We have to show that the set of elements of the form $E_{1}(\Delta) \hat{f}$, $f \in \mathfrak{S}$, is fundamental in $\mathfrak{R}^{2}(\rho)$. Let $\eta \in \mathfrak{R}^{2}(\rho)$ be such that $\left(\eta, E_{1}(\Delta) \hat{f}\right)=0$ for all intervals $\Delta$, and all $f \in \mathfrak{S}$ which are continuous and vanish outside compact subsets. We prove $\eta=0$, which implies the theorem. We have

$$
0=\left(\eta, E_{1}(\Delta) \hat{f}\right)=\int \sum_{\Delta, k=1}^{n}\left[\hat{f}_{k}(\lambda)\right]^{-} \eta_{j}(\lambda) d \rho_{j k}(\lambda),
$$

and this integral may be written as an inner product $\left(g_{\Delta}, f\right)$ in $\mathfrak{S}$ where

$$
g_{\Delta}(x)=\int_{\Delta} \sum_{j, k=1}^{n} s_{k}(x, \lambda) \eta_{j}(\lambda) d \rho_{j k}(\lambda)
$$

is an element in $\mathfrak{S}=\mathfrak{Q}^{2}(a, b)$. Indeed let $\delta$ be a finite subinterval of $(a, b)$, and let $h_{\delta}(x)=g_{\Delta}(x)$ for $x \in \delta$ and $h_{\delta}(x)=0$ otherwise. Then $h_{\delta} \in \mathfrak{S}$ and

$$
\begin{aligned}
\int_{\delta}\left|g_{\Delta}(x)\right|^{2} d x & =\int_{\delta} g_{\Delta}(x)\left[h_{\delta}(x)\right]-d x=\int_{\Delta} \sum_{j, k=1}^{n}\left[\hat{h}_{\delta k}(\lambda)\right]^{-} \eta_{j}(\lambda) d \rho_{j k}(\lambda) \\
& \leqq\left\|\hat{h}_{\delta}\right\|\|\eta\|=\left\|h_{\delta}\right\|\|\eta\|,
\end{aligned}
$$

using the Schwarz inequality and the isometry of $\mathfrak{S}$ onto $V \mathfrak{S}$. Thus 


$$
\int_{\delta}\left|g_{\Delta}(x)\right|^{2} d x \leqq\|\eta\|^{2}
$$

which shows that $g_{\Delta} \in \mathcal{R}^{2}(a, b)$, and $\left\|g_{\Delta}\right\| \leqq\|\eta\|$.

We now have $\left(g_{\Delta}, f\right)=0$ for a dense set of $f^{\prime}$ 's in $\mathfrak{S}$. Therefore $g_{\Delta}(x)=0$ almost everywhere, and since $g_{\Delta}$ is continuous, $g_{\Delta}(x)=0$ everywhere on $(a, b)$. Hence

$$
\begin{aligned}
g_{\Delta}^{(p-1)}(c) & =\int_{\Delta} \sum_{j, k=1}^{n} s_{k}^{(p-1)}(c, \lambda) \eta_{j}(\lambda) d \rho_{j k}(\lambda) \\
& =\int_{\Delta} \sum_{j=1}^{n} \eta_{j}(\lambda) d \rho_{j p}(\lambda)=0, \quad(p=1, \cdots, n) .
\end{aligned}
$$

Since this is valid for all finite intervals $\Delta$ we see that $(\zeta, \eta)=0$ for all vectors $\zeta \in \mathfrak{R}^{2}(\rho)$ whose components are step functions vanishing outside compact subsets of $(-\infty, \infty)$, and since these vectors are dense in $\mathfrak{l}^{2}(\rho)$, we have $\eta=0$ in $\mathfrak{R}^{2}(\rho)$ and the theorem is proved.

THEOREM 10. We have $V \mathfrak{S}=\mathfrak{R}^{2}(\rho)$ if and only if $E$ is a spectral function of $T_{0}$ which is a resolution of the identity of a self-adjoint extension $S$ of $T_{0}$ in $\mathfrak{E}$ itself.

Proof. First suppose $V \mathfrak{S}=\Omega^{2}(\rho)$. Let $S_{1}$ be the minimal self-adjoint extension of $\hat{T}_{0}$ of Theorem 9. Then $V E(\Delta) V^{-1}=P_{1} E_{1}(\Delta)=E_{1}(\Delta)$ on $V \mathfrak{S}=\mathfrak{Q}^{2}(\rho)$, since $P_{1}$ is the orthogonal projection of $\mathbb{R}^{2}(\rho)$ onto $V \mathfrak{S}=\Omega^{2}(\rho)$. Therefore $S=V^{-1} S_{1} V$ is a self-adjoint extension of $T_{0}$ in $\mathfrak{S}$ with the resolution of the identity $E$. Conversely, suppose $E$ is a resolution of the identity for a selfadjoint extension $S$ of $T_{0}$ in $\mathfrak{S}$. It is obviously a minimal one. Thus the operator $\hat{S}=V S V^{-1}$ is a minimal self-adjoint extension of $\hat{T}_{0}$ in $V \mathfrak{S E}$. It is the operator of multiplication by $\lambda$ on the set $\mathfrak{D}(\hat{S})=V \mathfrak{D}(S)$. However $S_{1}$ is also a minimal self-adjoint extension of $\hat{T}_{0}$, and $\hat{S} \subset S_{1}$. By Naimark's result $\hat{S}$ is unitarily equivalent to $S_{1}$. Therefore $V \mathfrak{S}=\Omega^{2}(\rho)$, and moreover $\hat{S}=S_{1}$.

Theorem 10 is the so-called inverse transform theorem, a different proof of which was given recently by Levinson [7].

In what follows we shall frequently identify $\mathfrak{S}$ with $V \mathfrak{S}, E$ with $V E V^{-1}$, and say that $S_{1}$ (of Theorem 9) is a minimal self-adjoint extension of $T_{0}$.

5. Ordinary differential operators on closed bounded intervals. In this section we assume the $n$th order ordinary differential operator $L=p_{0} D^{n}+\cdots$ $+p_{n}$ is given on a closed bounded interval $a \leqq x \leqq b$, that $p_{k} \in C^{n-k}$ there, and $p_{0}(x) \neq 0$ on $[a, b]$. We first compute in more detail the matrix $\Psi$ which determines the nature of a given generalized resolvent $R$ of $T_{0}$. As a convenience for our computations we shall choose the point $c$ to be $a$, and thus $s_{j}^{(k-1)}(a, l)$ $=\delta_{j k}$.

We recall some notations and results from [5]. The domain $\mathfrak{D}\left(T_{0}^{*}\right)$ is the set of all $u \in \mathfrak{S}=\mathfrak{R}^{2}(a, b)$ such that $u \in C^{n-1}$ on $[a, b], u^{(n-1)}$ is absolutely con- 
tinuous there, and $L u \in \mathfrak{S}$. For such $u, T_{0}^{*} u=L u$. For $u, v \in \mathfrak{D}\left(T_{0}^{*}\right)$ we have Green's formula

$$
\int_{y}^{x}(\bar{v} L u-u \overline{L v}) d t=[u v](x)-[u v](y)
$$

where $[u v](x)$ is a form in $\left(u, u^{\prime}, \cdots, u^{(n-1)}\right)$ and $\left(v, v^{\prime}, \cdots, v^{(n-1)}\right)$ which we write as

$$
[u v](x)=\sum_{j, k=1}^{n} B_{j k}^{0}(x) u^{(k-1)}(x)\left[v^{(j-1)}(x)\right]^{-} .
$$

The matrix $B_{0}(x)=\left(B_{j k}^{0}(x)\right)$ is skew-hermitian, and its elements are linear combinations, with constant coefficients, of the coefficients in $L$. For $u, v$ $\in \mathfrak{D}\left(T_{0}^{*}\right)$ we let $\langle u v\rangle=(L u, v)-(u, L v)$, which by Green's formula is equal to $[u v](b)-[u v](a)$.

The set $R$ of all generalized resolvents of $T_{0}$ are in a one-to-one correspondence with the set $\mathcal{F}$ of all operator-valued functions $F$ defined on $\operatorname{Im} l>0$ which take $\mathbb{E}(-i)$ into $\mathbb{E}(i)$, are analytic on $\operatorname{Im} l>0$, and such that $\|F(l)\| \leqq 1$. In the case under consideration $\operatorname{dim} \xi(i)=\operatorname{dim}(\xi(-i)=n$. Let $\phi_{1}, \cdots, \phi_{n}$ and $\psi_{1}, \cdots, \psi_{n}$ be orthonormal bases for $\&(i)$ and $\&(-i)$ respectively. If $F \in \mathcal{F}$, define the functions $v_{j}(l), v_{j}^{*}(l)$ by

$$
\begin{array}{ll}
v_{j}(l) & =\psi_{j}-F(l) \psi_{j}, \\
v_{j}^{*}(l) & =\phi_{j}-F^{*}(l) \phi_{j},
\end{array}
$$

If $R \in R$ there is a unique $F \in \mathcal{F}$ such that the range of $R(l)$ for $\operatorname{Im} l>0$ is the set of all $u \in \mathfrak{D}\left(T_{0}^{*}\right)$ satisfying

$$
\left\langle u v_{j}^{*}(l)\right\rangle=0, \quad(j=1, \cdots, n),
$$

and the range of $R(\bar{l})$ is the set of all $u \in \mathfrak{D}\left(T_{0}^{*}\right)$ satisfying

$$
\left\langle u v_{j}(l)\right\rangle=0, \quad(j=1, \cdots, n) ;
$$

see Theorem 1 of [5]. Every $F \in \mathcal{F}$ appears in this process.

Let $R$ be a fixed generalized resolvent of $T_{0}$ and let $F \in \mathcal{F}$ correspond to it. We write $R(l)=R_{0}(l)+R_{1}(l)$, where $R_{0}(l), R_{1}(l)$ are integral operators with kernels $K_{0}, K_{1}$ respectively. The kernel $K_{0}$ is given explicitly by

$$
K_{0}(x, y, l)=\left[K_{0}(y, x, \bar{l})\right]^{-}=\frac{1}{2} \sum_{j, k=1}^{n} S_{j k}^{-1} s_{k}(x, l)\left[s_{j}(y, \bar{l})\right]^{-}, \quad(x \geqq y),
$$

where $L s_{j}(x, l)=l s_{j}(x, l), s_{j}^{(k-1)}(a, l)=\delta_{j k}$, and $S_{j k}=\left[s_{j}(l) s_{k}(\bar{l})\right]$, which is independent of $l$. The matrix $S=\left(S_{j k}\right)$ is nonsingular, skew-hermitian, and $S_{\mathbf{k}}^{-1}$ is the element in the $j$ th row and $k$ th column of $S^{-1}$, i.e. $S^{-1}=\left(S_{j k}^{-1}\right)$. We recall that $K_{1}$ is given by 


$$
K_{1}(x, y, l)=\sum_{j, k=1}^{n} \Psi_{j k}(l) s_{k}(x, l)\left[s_{j}(y, \bar{l})\right]^{-} .
$$

Let $\operatorname{Im} l>0$ and $f \in \mathfrak{G}$. Then we have $R(l) f=R_{0}(l) f+R_{1}(l) f$ where

(5.2) $R_{0}(l) f(x)=\frac{1}{2} \sum_{j, k=1}^{n} S_{j k}^{-1} s_{k}(x, l)\left[\int_{a}^{x}\left[s_{j}(y, \bar{l})\right]-f(y) d y-\int_{x}^{b}\left[s_{j}(y, \bar{l})\right]-f(y) d y\right]$ and

$$
R_{1}(l) f(x)=\sum_{j, k=1}^{n} \Psi_{j k}(l)\left(f, s_{j}(\bar{l})\right) s_{k}(x, l) .
$$

The conditions $\left\langle R(l) f v_{p}^{*}(l)\right\rangle=0, p=1, \cdots, n$, imply

$$
\left\langle R_{0}(l) f v_{p}^{*}(l)\right\rangle+\sum_{j, k=1}^{n} \Psi_{j k}(l)\left(f, s_{j}(\bar{l})\right)\left\langle s_{k}(l) v_{p}^{*}(l)\right\rangle=0 .
$$

Let $V(l)=\left(V_{k p}(l)\right)=\left(\left\langle s_{k}(l) v_{p}^{*}(l)\right\rangle\right)$. This matrix is nonsingular, since if $c_{1}, \cdots, c_{n}$ are constants such that $\sum c_{k}\left\langle s_{k}(l) v_{p}^{*}(l)\right\rangle=0$ then the function $u(l)=\sum c_{k} s_{k}(l)$ is in the range of $R(l)$ and in $\&(l)$. Thus there is a $g \in \mathfrak{S}$ such that $R(l) g=u$, and $\left(T_{0}^{*}-l\right) R(l) g=\left(T_{0}^{*}-l\right) u=0$, and since $R(l)$ is a right inverse of $\left(T_{0}^{*}-l\right)$ we have $g=0$ and hence $u=0$. If $V^{-1}(l)=\left(V_{p \boldsymbol{q}}^{-1}(l)\right)$, multiplying (5.3) by $V_{p q}^{-1}(l)$ and summing on $p$ yields

$$
\sum_{p=1}^{n}\left\langle R_{0}(l) f v_{p}^{*}(l)\right\rangle V_{p q}^{-1}(l)+\sum_{j=1}^{n} \Psi_{j q}(l)\left(f, s_{j}(\bar{l})\right)=0 .
$$

From (5.2) it follows that

$$
\left\langle R_{0}(l) f v_{p}^{*}(l)\right\rangle=\frac{1}{2} \sum_{j, k=1}^{n} S_{j k}^{-1}\left\{\left[s_{k}(l) v_{p}^{*}(l)\right](b)+\left[s_{k}(l) v_{p}^{*}(l)\right](a)\right\}\left(f, s_{j}(\bar{l})\right) .
$$

Placing this into (5.4), using the fact that this is valid for all $f \in \mathfrak{S}$, and the fact that the $s_{j}(\bar{l})$ are linearly independent, we obtain

$$
\Psi_{j q}(l)=-\frac{1}{2} \sum_{k, p=1}^{n} S_{j k}^{-1}\left\{\left[s_{k}(l) v_{p}^{*}(l)\right](b)+\left[s_{k}(l) v_{p}^{*}(l)\right](a)\right\} V_{p q}^{-1}(l) .
$$

Let $Q(x, l)=\left(Q_{k p}(x, l)\right)=\left(\left[s_{k}(l) v_{p}^{*}(l)\right](x)\right)$. Then $V(l)=Q(b, l)-Q(a, l)$, and we have

$$
\Psi(l)=-\frac{1}{2} S^{-1}[Q(b, l)+Q(a, l)][Q(b, l)-Q(a, l)]^{-1} .
$$

From (5.1) we see that

$$
Q_{k p}(x, l)=\left[s_{k}(l) v_{p}^{*}(l)\right](x)=\sum_{\alpha, \beta=1}^{n} B_{\alpha \beta}^{0}(x) s_{k}^{(\beta-1)}(x, l)\left[v_{p}^{*}(\alpha-1)(x, l)\right]^{-} .
$$


Let $S(x, l)=\left(S_{\beta k}(x, l)\right)=\left(s_{\boldsymbol{z}}^{(\beta-1)}(x, l)\right)$ and $W(x, l)=\left(W_{\alpha p}(x, l)\right)=\left(v_{p}^{*(\alpha-1)}(x, l)\right.$. Then from (5.6) we find

$$
Q^{t}(x, l)=W^{*}(x, l) B_{0}(x) S(x, l),
$$

where $Q^{t}$ is the transposed matrix of $Q$.

We place in evidence the dependence of $\Psi$ on $F$. Let

$$
F(l) \psi_{j}=\sum_{p=1}^{n} F_{p j}(l) \phi_{p}
$$

and identify $F(l)$ with the matrix with $F_{p j}(l)$ in the $p$ th row and $j$ th column, $F(l)=\left(F_{p j}(l)\right)$. This matrix is analytic for $\operatorname{Im} l>0$ and $\|F(l)\| \leqq 1$, where

$$
\|F(l)\|=\sup \|F(l) \xi\|, \quad\|\xi\|=1,
$$

and $\xi$ is an $n$-dimensional column vector of complex numbers. Now

$$
F^{*}(l) \phi_{p}=\sum_{q=1}^{n}\left[F_{p q}(l)\right]-\psi_{q},
$$

and hence

$$
W_{\alpha p}(x, l)=v_{p}^{*(\alpha-1)}(x, l)=\phi_{p}^{(\alpha-1)}(x)-\sum_{q=1}^{n}\left[F_{p q}(l)\right]-\psi_{q}^{(\alpha-1)}(x) .
$$

If $\phi(x)=\left(\phi_{\alpha p}(x)\right)=\left(\phi_{p}^{(\alpha-1)}(x)\right)$ and $\psi(x)=\left(\psi_{\alpha q}(x)\right)=\left(\psi_{q}^{(\alpha-1)}(x)\right)$, then we see that $W(x, l)=\phi(x)-\psi(x) F^{*}(l)$, and thus $W^{*}(x, l)=\phi^{*}(x)-F(l) \psi^{*}(x)$. Placing this into (5.7) we obtain

$$
Q^{t}(x, l)=\phi^{*}(x) B_{0}(x) S(x, l)-F(l) \psi^{*}(x) B_{0}(x) S(x, l),
$$

and the following theorem results from (5.5).

THEOREM 11. Let $L$ be defined on a closed bounded interval $[a, b]$, and let $\Psi \in \mathfrak{T}$. Then

$$
\Psi^{t}(l)=-\frac{1}{2}[C(l)-F(l) D(l)]^{-1}[A(l)-F(l) B(l)]\left(S^{t}\right)^{-1}
$$

for $\operatorname{Im} l>0$, where $A, B, C, D$ are matrices of entire functions, depending only on $L$, given by

$$
\begin{aligned}
& A(l)=\phi^{*}(b) B_{0}(b) S(b, l)+\phi^{*}(a) B_{0}(a) S(a, l), \\
& B(l)=\psi^{*}(b) B_{0}(b) S(b, l)+\psi^{*}(a) B_{0}(a) S(a, l), \\
& C(l)=\phi^{*}(b) B_{0}(b) S(b, l)-\phi^{*}(a) B_{0}(a) S(a, l), \\
& D(l)=\psi^{*}(b) B_{0}(b) S(b, l)-\psi^{*}(a) B_{0}(a) S(a, l),
\end{aligned}
$$

and $F$ is an $n$ by $n$ matrix which is analytic for $\operatorname{Im} l>0,\|F(l)\| \leqq 1$ (operator norm). If $F$ is any matrix of this type the $\Psi$ defined via (5.9) will be in $\mathfrak{T}$. 
Using Theorem 11 we give a qualitative description of a large number of $\Psi$ 's and corresponding $\rho$ 's.

TheOREM 12. Suppose $F$ is continuous on $\operatorname{Im} l \geqq 0$ and

$$
\sup _{\operatorname{Im} l>0}\|F(l)\|=r<1 .
$$

Then $\rho$ is absolutely continuous with respect to Lebesgue measure on $(-\infty, \infty)$, and has a continuous positive definite density.

Proof. We have

$$
\rho(\lambda)=\lim _{\epsilon \rightarrow+0} \frac{1}{\pi} \int_{0}^{\lambda} \operatorname{Im} \Psi(\nu+i \epsilon) d \nu .
$$

Since $\Psi^{t}$ will be a little more convenient to work with, we compute $\operatorname{Im} \Psi^{t}$. From (5.5) we have

$$
\Psi^{t}(l)=-\frac{1}{2}[P(b, l)-P(a, l)]^{-1}[P(b, l)+P(a, l)]\left(S^{t}\right)^{-1},
$$

where $P(x, l)=Q^{t}(x, l)$. A short calculation then gives

$$
\operatorname{Im} \Psi^{t}(l)=[P(b, l)-P(a, l)]^{-1} \chi(l)[P(b, l)-P(a, l)]^{*-1},
$$

where

$$
2 i \chi(l)=P(a, l)\left(S^{t}\right)^{-1} P^{*}(a, l)-P(b, l)\left(S^{t}\right)^{-1} P^{*}(b, l) .
$$

We claim that $\chi(l)$ may be written as

$$
\chi(l)=\left[I-F(l) F^{*}(l)\right]+\Omega(l),
$$

where

$$
-2 i \Omega(l)=W^{*}(b, l) B_{0}(b) W(b, l)+P(b, l)\left(S^{t}\right)^{-1} P^{*}(b, l),
$$

and $I$ is the identity matrix. To prove this we require several identities. Since $S_{\alpha \beta}=\left[s_{\alpha}(l) s_{\beta}(\bar{l})\right](x)$, which is independent of $x$ and $l$, we have from (5.1)

(5.15) $S^{t}=S^{*}(x, \bar{l}) B_{0}(x) S(x, l), \quad\left(S^{t}\right)^{-1}=S^{-1}(x, l) B_{0}^{-1}(x) S^{*-1}(x, \bar{l})$.

Also since $L \phi_{\alpha}=i \phi_{\alpha}, L \psi_{\alpha}=-i \psi_{\alpha},\left(\phi_{\alpha}, \phi_{\beta}\right)=\left(\psi_{\alpha}, \psi_{\beta}\right)=\delta_{\alpha \beta}$, we have

$$
\left[\phi_{\alpha} \phi_{\beta}\right](b)-\left[\phi_{\alpha} \phi_{\beta}\right](a)=\left(L \phi_{\alpha}, \phi_{\beta}\right)-\left(\phi_{\alpha}, L \phi_{\beta}\right)=2 i \delta_{\alpha \beta},
$$

and hence

$$
\phi^{*}(b) B_{0}(b) \phi(b)-\phi^{*}(a) B_{0}(a) \phi(a)=2 i I
$$

Similarly

$$
\begin{aligned}
& \psi^{*}(b) B_{0}(b) \psi(b)-\psi^{*}(a) B_{0}(a) \psi(a)=-2 i I \\
& \psi^{*}(b) B_{0}(b) \phi(b)-\psi^{*}(a) B_{0}(a) \phi(a)=0 .
\end{aligned}
$$


We note that since $s_{f}^{(k-1)}(a, l)=\delta_{j k}$ we have $S(a, l)=I$. Using (5.15) at $x=a$ we obtain $\left(S^{t}\right)^{-1}=B_{0}^{-1}(a)$, and hence

$$
\begin{aligned}
P(a, l)\left(S^{t}\right)^{-1} P^{*}(a, l) & =W^{*}(a, l) B_{0}(a) S(a, l)\left(S^{t}\right)^{-1} S^{*}(a, l) B_{0}^{*}(a) W(a, l) \\
& =-W^{*}(a, l) B_{0}(a) W(a, l),
\end{aligned}
$$

since $B_{0}^{*}(x)=-B_{0}(x)$. Now $W^{*}(x, l)=\phi^{*}(x)-F(l) \psi^{*}(x)$, and by making use of (5.16) and (5.17) we obtain

$$
\begin{aligned}
P(a, l)\left(S^{t}\right)^{-1} P^{*}(a, l)= & -W^{*}(b, l) B_{0}(b) W(b, l) \\
& +2 i\left[I-F(l) F^{*}(l)\right] .
\end{aligned}
$$

Placing this into (5.12) yields (5.13).

Since sup $\|F(l)\|=r<1, \operatorname{Im} l>0$, we have $I-F(l) F^{*}(l) \geqq\left(1-r^{2}\right) I>0$. We show that $\Omega(l) \geqq 0$. Let $T(\bar{l})$ be the matrix with $\left(s_{j}(\bar{l}), s_{k}(\bar{l})\right)$ as element in the $j$ th row and $k$ th column. Since $T(\bar{l})$ is the Gramian matrix of the basis $s_{1}(\bar{l}), \cdots, s_{n}(\bar{l})$ we see that $T(\bar{l})>0$, and hence $T^{t}(\bar{l})>0$. We prove that

$$
\Omega(l)=\operatorname{Im} l P(b, l)\left(S^{t}\right)^{-1} T^{t}(\bar{l})\left(S^{*}\right)^{-1} P^{*}(b, l),
$$

which is non-negative for $\operatorname{Im} l>0$. Now

$$
\begin{aligned}
{\left[s_{j}(\bar{l}) s_{k}(\bar{l})\right](b)-\left[s_{j}(\bar{l}) s_{k}(\bar{l})\right](a) } & =\left(L s_{j}(\bar{l}), s_{k}(\bar{l})\right)-\left(s_{j}(\bar{l}), L s_{k}(\bar{l})\right) \\
& =-2 i \operatorname{Im} l\left(s_{j}(\bar{l}), s_{k}(\bar{l})\right),
\end{aligned}
$$

and hence

$$
-2 i \operatorname{Im} l T^{t}(\bar{l})=S^{*}(b, \bar{l}) B_{0}(b) S(b, \bar{l})-S^{*}(a, \bar{l}) B_{0}(a) S(a, \bar{l}) .
$$

Therefore

$$
-2 i \operatorname{Im} l P(b, l)\left(S^{t}\right)^{-1} T^{t}(\bar{l})\left(S^{*}\right)^{-1} P^{*}(b, l)=(\alpha)+(\beta),
$$

where

$$
\begin{aligned}
(\alpha) & =P(b, l)\left(S^{t}\right)^{-1} S^{*}(b, \bar{l}) B_{0}(b) S(b, \bar{l})\left(S^{*}\right)^{-1} P^{*}(b, l) \\
& =W^{*}(b, l) B_{0}(b) W(b, l)
\end{aligned}
$$

using (5.7) and (5.15). Now

$$
\begin{aligned}
(\beta) & =-P(b, l)\left(S^{t}\right)^{-1} S^{*}(a, \bar{l}) B_{0}(a) S(a, \bar{l})\left(S^{*}\right)^{-1} P^{*}(b, l) \\
& =P(b, l)\left(S^{t}\right)^{-1} P^{*}(b, l),
\end{aligned}
$$

making use of $S(a, l)=S(a, \bar{l})=I$ and $\left(S^{t *}\right)^{-1}=-\left(S^{t}\right)^{-1}=-B_{0}^{-1}(a)$. From (5.19) and the expressions developed for $(\alpha)$ and $(\beta)$ we see that (5.18) is valid; see (5.14).

Returning to (5.11)-(5.13) we see that since $F$ is continuous for $\operatorname{Im} l \geqq 0$, $P(x, l)=Q^{t}(x, l)$ tends to a limit as $\operatorname{Im} l \rightarrow 0$. Thus $\chi(l)$ and $P(b, l)-P(a, l)$ tend to limits as $\operatorname{Im} l \rightarrow 0$. Moreover we have from (5.13) 


$$
\chi(l) \geqq\left(1-r^{2}\right) I
$$

$\operatorname{Im} l>0$,

which shows that

$$
\chi(\nu)=\lim _{\epsilon \rightarrow+0} \chi(\nu+i \epsilon) \geqq\left(1-r^{2}\right) I>0 .
$$

We claim that $[P(b, \nu)-P(a, \nu)]^{-1}$ exists for $\nu$ real. For if it did not exist there would be a column vector $\eta \neq 0$ such that $P^{*}(b, \nu) \eta=P^{*}(a, \nu) \eta$ and $\eta^{*} P(b, \nu)=\eta^{*} P(a, \nu)$. Thus

$$
\eta^{*} P(b, \nu)\left(S^{t}\right)^{-1} P^{*}(b, \nu) \eta=\eta^{*} P(a, \nu)\left(S^{t}\right)^{-1} P^{*}(a, \nu) \eta,
$$

which implies by (5.12) $\eta^{*} \chi(\nu) \eta=0$. But this contradicts the fact that $\chi(\nu)>0$. Hence $[P(b, \nu)-P(a, \nu)]^{-1}$ exists for all real $\nu$. From (5.18) it is clear that

$$
\lim _{\epsilon \rightarrow+0} \Omega(\nu+i \epsilon)=0,
$$

and therefore we obtain from (5.11) and (5.13)

$$
\operatorname{Im} \Psi^{t}(\nu)=[P(b, \nu)-P(a, \nu)]^{-1}\left[I-F(\nu) F^{*}(\nu)\right][P(b, \nu)-P(a, \nu)]^{*-1},
$$

or

$$
\operatorname{Im} \Psi(\nu)=[Q(b, \nu)-Q(a, \nu)]^{*-1}\left[I-F(\nu) F^{*}(\nu)\right]^{t}[Q(b, \nu)-Q(a, \nu)]^{-1},
$$

which is clearly continuous and positive definite. Since $\operatorname{Im} \Psi$ is uniformly continuous on any rectangle of the form $0 \leqq \nu \leqq \lambda, 0 \leqq \epsilon \leqq \delta$, we have

$$
\rho(\lambda)=\lim _{\epsilon \rightarrow+0} \frac{1}{\pi} \int_{0}^{\lambda} \operatorname{Im} \Psi(\nu+i \epsilon) d \nu=\frac{1}{\pi} \int_{0}^{\lambda} \operatorname{Im} \Psi(\nu) d \nu,
$$

which completes the proof of the theorem.

Before exploiting the result of Theorem 12 we discuss the geometry of the set $\mathscr{N}$ of all the $\Psi^{\prime}$ 's. From the expression for $\Psi^{t}(l)$ just above (5.11) we see that

$$
\Psi^{t}(l)-\frac{1}{2}\left(S^{t}\right)^{-1}=-[P(b, l)-P(a, l)]^{-1} P(b, l)\left(S^{t}\right)^{-1},
$$

which gives by (5.18)

$$
\begin{aligned}
& {[P(b, l)-P(a, l)]^{-1} \Omega(l) } {[P(b, l)-P(a, l)]^{*-1} } \\
&=\operatorname{Im} l\left[\Psi^{t}(l)-\frac{1}{2}\left(S^{t}\right)^{-1}\right] T^{t}(\bar{l})\left[\Psi^{t}(l)-\frac{1}{2}\left(S^{t}\right)^{-1}\right]^{*} .
\end{aligned}
$$

From (5.11) and (5.13) therefore it follows that

$$
\begin{aligned}
\operatorname{Im} \Psi(l)=[ & Q(b, l)-Q(a, l)]^{*-1}\left[I-F(l) F^{*}(l)\right]^{t}[Q(b, l)-Q(a, l)]^{-1} \\
& +\operatorname{Im} l\left[\Psi(l)-\frac{1}{2} S^{-1}\right]^{*} T(\bar{l})\left[\Psi(l)-\frac{1}{2} S^{-1}\right] .
\end{aligned}
$$


Thus

$$
\operatorname{Im} \Psi(l) \geqq \operatorname{Im} l\left[\Psi(l)-\frac{1}{2} S^{-1}\right]^{*} T(\bar{l})\left[\Psi(l)-\frac{1}{2} S^{-1}\right],
$$

and equality holds if and only if $F(l) F^{*}(l)=I$, i.e. $F(l)$ is unitary. If $F\left(l_{0}\right)$ is unitary for some $l_{0}, \operatorname{Im} l_{0}>0$, it is unitary for all $l$, and $F(l)=F\left(l_{0}\right)$ for $\operatorname{Im} l>0$. Indeed if $F\left(l_{0}\right)$ is unitary $\left\|F\left(l_{0}\right) \xi\right\|=\|\xi\|$ for every $n$-dimensional vector $\xi$. Consider the analytic function $f(l)=\left(F(l) \xi, \quad F\left(l_{0}\right) \xi\right)$. We have $|f(l)|$ $\leqq\|F(l) \xi\|\left\|F\left(l_{0}\right) \xi\right\| \leqq\|\xi\|^{2}$, and $\left|f\left(l_{0}\right)\right|=\left\|F\left(l_{0}\right) \xi\right\|^{2}=\|\xi\|^{2}$. By the maximum modulus theorem $f(l)=\|\xi\|^{2}$ for $\operatorname{Im} l>0$. Thus $\left(F(l) \xi, F\left(l_{0}\right) \xi\right)=(\xi, \xi)$ for all $\xi$, which implies $F^{*}\left(l_{0}\right) F(l)=I$, and hence $F(l)=F\left(l_{0}\right)$. It now follows that we have equality in (5.20) for all $l, \operatorname{Im} l>0$, or we have a strict inequality for all $l$, Im $l>0$. Equality occurs if and only if $F$ is a constant unitary matrix. A glance at the way in which the matrix $F$ arises (see [5]) shows that $F$ is constant unitary if and only if the corresponding generalized resolvent $R$ is a resolvent of a self-adjoint extension of $T_{0}$ in $\mathfrak{E}=\mathfrak{R}^{2}(a, b)$ itself.

We interpret the inequality (5.20) geometrically. Noting that $\operatorname{Im} \Psi$ $=(1 / 2 i)\left(\Psi-\Psi^{*}\right)$, we may rewrite $(5.20)$ as follows

$$
\begin{aligned}
\Psi^{*}(l) T(\bar{l}) \Psi(l)-\left[S_{0}^{*} T(\bar{l})\right. & \left.+\frac{I}{2 i \operatorname{Im} l}\right] \Psi(l) \\
& -\Psi^{*}(l)\left[T(\bar{l}) S_{0}-\frac{I}{2 i \operatorname{Im} l}\right]+S_{0}^{*} T(\bar{l}) S_{0} \leqq 0,
\end{aligned}
$$

where $S_{0}=(1 / 2) S^{-1}$. Let $T_{0}^{1 / 2}(\bar{l})$ denote the positive square root of $T(\bar{l})$, and let

$$
\Lambda(l)=T^{1 / 2}(\bar{l}) \Psi(l), \quad \Lambda_{0}(l)=T^{-1 / 2}(\bar{l})\left[T(\bar{l}) S_{0}-\frac{I}{2 i \operatorname{Im} l}\right]
$$

In terms of these matrices (5.21) becomes

$$
\left[\Lambda(l)-\Lambda_{0}(l)\right]^{*}\left[\Lambda(l)-\Lambda_{0}(l)\right] \leqq M_{0}(l)
$$

where

$$
M_{0}(l)=\frac{S^{-1}}{2 i \operatorname{Im} l}+\frac{T^{-1}(\bar{l})}{4(\operatorname{Im} l)^{2}} .
$$

THEOREM 13. For each $l, \operatorname{Im} l>0$, and $\Psi \in \mathfrak{T}$, the matrix $\Lambda(l)=T^{1 / 2}(\bar{l}) \Psi(l)$ lies inside or on the "circle" (5.22) with "center" $\Lambda_{0}(l)$ and "radius" $M_{0}^{1 / 2}(l)$. If $\Lambda(l)$ is on the "circumference" of this circle for one lit is on the "circumference" for all $l, \operatorname{Im} l>0$, and this occurs if and only if $\Psi$ corresponds to a generalized resolvent of a self-adjoint extension of $T_{0}$ in $\mathfrak{R}^{2}(a, b)$ itself.

We return to the implication of Theorem 12 . 
THEOREM 14. Let $F$ satisfy the same conditions as in Theorem 12. Then the minimal self-adjoint extension $S_{1}$ (see Theorem 9) of $T_{0}$ is unitarily equivalent to the operator $i D \oplus \cdots \oplus i D$ (n-times) on $\mathbb{R}^{2}(-\infty, \infty) \oplus \cdots \oplus \mathbb{R}^{2}(-\infty, \infty)$ (n times).

Proof. The domain $\mathfrak{D}(i D)$ is the set of all $u \in \mathbb{R}^{2}(-\infty, \infty)$ which are absolutely continuous on $(-\infty, \infty)$ and such that $i u^{\prime} \in \mathbb{R}^{2}(-\infty, \infty)$. For such $u, i D u(x)=i u^{\prime}(x)$. This operator is self-adjoint in $\mathfrak{R}^{2}(-\infty, \infty)$.

According to Theorem 12 the $\rho$ corresponding to $F$ is given by

$$
\rho(\lambda)=\frac{1}{\pi} \int_{0}^{\lambda} \operatorname{Im} \Psi(\nu) d \nu,
$$

where $N^{t}(\nu)=(1 / \pi) \operatorname{Im} \Psi(\nu)$ is continuous and positive definite. Let $U$ be the mapping of $\mathfrak{R}^{2}(\rho)$ into $\mathfrak{R}_{n}^{2}(-\infty, \infty)=\mathfrak{R}^{2}(-\infty, \infty) \oplus \cdots \oplus \mathfrak{R}^{2}(-\infty, \infty)$ ( $n$ times) given by $U \zeta(\lambda)=N^{1 / 2}(\lambda) \zeta(\lambda)$, where $N^{1 / 2}(\lambda)$ is the positive square root of $N(\lambda)$. Then $U$ is a unitary map of $\mathfrak{R}^{2}(\rho)$ onto $\mathfrak{R}_{n}^{2}(-\infty, \infty)$. Indeed we have $d \rho(\lambda)=N^{t}(\lambda) d \lambda$ and thus the inner product on $\mathfrak{R}^{2}(\rho)$ is given by

$$
(\zeta, \eta)=\int_{-\infty}^{\infty}(N(\lambda) \zeta(\lambda), \eta(\lambda)) d \lambda
$$

where the inner product under the integral sign is the usual one for complex $n$-dimensional vectors. The inner product in $\mathfrak{R}_{n}^{2}(-\infty, \infty)$ is given by

$$
(\alpha, \beta)_{n}=\int_{-\infty}^{\infty}(\alpha(\lambda), \beta(\lambda)) d \lambda .
$$

Therefore if $\zeta \in \mathfrak{R}^{2}(\rho)$ and $U \zeta=N^{1 / 2} \zeta$ we have

$$
\begin{aligned}
\|\zeta\|^{2} & =\int_{-\infty}^{\infty}(N(\lambda) \zeta(\lambda), \zeta(\lambda)) d \lambda=\int_{-\infty}^{\infty}\left(N^{1 / 2}(\lambda) \zeta(\lambda), N^{1 / 2}(\lambda) \zeta(\lambda)\right) d \lambda \\
& =\int_{-\infty}^{\infty}(U \zeta(\lambda), U \zeta(\lambda)) d \lambda=\|U \zeta\|_{n}^{2},
\end{aligned}
$$

which shows that $U$ is an isometry. It is onto since $U^{-1} \alpha=N^{-1 / 2} \alpha$ for all $\alpha \in \mathbb{R}_{n}^{2}(-\infty, \infty)$.

The operator $S_{1}$ has a domain $\mathfrak{D}\left(S_{1}\right)$ consisting of all $\zeta \in \mathbb{R}^{2}(\rho)$ such that $\lambda \zeta \in \mathbb{R}^{2}(\rho)$, and for such $\zeta, S_{1} \zeta(\lambda)=\lambda \zeta(\lambda)$. Let $\Sigma_{1}$ be the operator of multiplication by $\lambda$ on $\mathfrak{R}_{n}^{2}(-\infty, \infty)$. We have $\mathfrak{D}\left(\Sigma_{1}\right)$ is the set of all $\alpha \in \mathfrak{R}_{n}^{2}(-\infty, \infty)$ such that $\lambda \alpha \in \mathbb{R}_{n}^{2}(-\infty, \infty)$ and for such $\alpha, \Sigma_{1} \alpha(\lambda)=\lambda \alpha(\lambda)$. Since for $\zeta \in \mathfrak{D}\left(S_{1}\right)$, $U \lambda \zeta=N^{1 / 2} \lambda \zeta=\lambda U \zeta$, we see that $U \mathfrak{D}\left(S_{1}\right)=\mathfrak{D}\left(\Sigma_{1}\right)$, and moreover $\Sigma_{1} U \zeta=U S_{1} \zeta$ for all $\zeta \in \mathfrak{D}\left(S_{1}\right)$. Hence $\Sigma_{1}=U S_{1} U^{-1}$. However $\Sigma_{1}$ is unitarily equivalent to $i D \oplus \cdots \oplus i D$ ( $n$ times) on $\ell_{n}^{2}(-\infty, \infty)$ by the Fourier transform theorem. This completes the proof of Theorem 14. 
6. An approximation result. Suppose $R$ is a generalized resolvent of $T_{0}$, where $L$ is now defined on some open interval $(a, b)$. According to A. V. Straus [10]

$$
R(l)=\left(T_{F(l)}-l I\right)^{-1},
$$

where $T_{F(l)}$ is such that $T_{0} \subset T_{F(l)} \subset T_{0}^{*}$, and $\mathfrak{D}\left(T_{F(l)}\right)$ is the set of all $u \in \mathfrak{D}\left(T_{0}^{*}\right)$ of the form

$$
u=u_{0}+(I-F(l)) u^{-}, \quad u_{0} \in \mathfrak{D}\left(T_{0}\right), \quad u^{-} \in \mathbb{E}(-i) .
$$

Here $F$ is a unique operator-valued function taking $\&(-i)$ into $\&(i)$ which is analytic for $\operatorname{Im} l>0$ and $\|F(l)\| \leqq 1$. The domain of $T_{F(l)}$, which is the range of $R(l)$, is the set of all $u \in \mathfrak{D}\left(T_{0}^{*}\right)$ satisfying the boundary conditions

$$
\left\langle u v_{j}^{*}(l)\right\rangle=0, \quad\left(j=1, \cdots, \omega^{+}\right) .
$$

Here we set $\omega^{+}=\operatorname{dim}\left(E(i)\right.$, and if $\phi_{1}, \cdots, \phi_{\omega^{+}}$is an orthonormal basis for (5) $(i)$

$$
v_{j}^{*}(l)=\phi_{j}-F^{*}(l) \phi_{j}, \quad\left(j=1, \cdots, \omega^{+}\right)
$$

see $[5]$.

Let $\delta=\left[\begin{array}{ll}\tilde{a}, & \tilde{b}\end{array}\right]$ be any closed bounded subinterval of $(a, b)$, and let $\phi_{1 \delta}, \cdots, \phi_{\omega^{+} \delta}$ be $\phi_{1}, \cdots, \phi_{\omega^{+}}$orthonormalized in $\mathfrak{l}^{2}(\delta)$. Let

$$
v_{j \delta}^{*}(l)=\phi_{j \delta}-F^{*}(l) \phi_{j \delta}, \quad \quad\left(j=1, \cdots, \omega^{+}\right) .
$$

Let $\phi_{\omega^{+}+1} \delta, \cdots, \phi_{n \delta}$ be functions such that $\phi_{1 \delta}, \cdots, \phi_{n \delta}$ is an orthonormal basis in $\mathfrak{R}^{2}(\delta)$ for the solutions of $L u=i u$, and let

$$
v_{j \delta}^{*}(l)=\phi_{j \delta}, \quad\left(j=\omega^{+}+1, \cdots, n\right) .
$$

If

$$
F_{\gamma}(l)=\left(\begin{array}{cc}
F(l) & 0 \\
0 & 0
\end{array}\right)
$$

we see that

$$
\stackrel{*}{v_{j \delta}(l)}=\phi_{j \delta}-F_{\delta}^{*}(l) \phi_{j \delta}, \quad(j=1, \cdots, n) .
$$

Clearly $F_{\delta}$ is analytic for $\operatorname{Im} l>0$, and $\left\|F_{\delta}(l)\right\| \leqq 1$. Thus $F_{\delta}$ gives rise to a generalized resolvent $R_{\delta}$ of the minimal operator $T_{0 \delta}$ associated with $L$ on $\mathfrak{l}^{2}(\delta)$, and the range of $R_{\delta}(l)$ is the set of all $u \in \mathfrak{D}\left(T_{0 \delta}^{*}\right)$ satisfying

$$
\left\langle u v_{j \delta}^{*}(l)\right\rangle=0, \quad(j=1, \cdots, n) .
$$

We have $v_{j \delta}^{*}(l) \rightarrow v_{j}^{*}(l)\left(j=1, \cdots, \omega^{+}\right)$in the pointwise sense as well as in $\mathfrak{R}^{2}(a, b)$. It now follows that the results of [3] carry over to the above situation. If $K_{\delta}, K$ are the kernels of $R_{\delta}, R$ respectively, then $K_{\delta} \rightarrow K$ uniformly on 
any compact $(x, y, l)$-region where $\operatorname{Im} l \neq 0$. This implies that $\Psi_{\delta} \rightarrow \Psi$ uniformly on compact subsets of $\pi$. Appealing to the lemma of $\S 3$ and the first part of the proof of Theorem 7, we obtain

$$
\rho_{\delta}(\lambda)-\rho_{\delta}(\mu) \rightarrow \rho(\lambda)-\rho(\mu)
$$

at continuity points $\lambda, \mu$ of $\rho$. We omit the details, referring the reader to the reasoning given in $[3]$.

\section{REFERENCES}

1. N. I. Achieser and I. M. Glasmann, Theorie der linearen Operatoren im Hilbert-Raum, Akademie Verlag, Berlin, 1954 (German translation).

2. N. Bourbaki, Espaces vectoriels topologiques, Eléments de Mathématique, A. S. I. 1189, Hermann et Cie, Paris, 1953.

3. E. A. Coddington, On maximal symmetric ordinary differential operators, Math. Scand. vol. 4 (1956) pp. 22-28.

4. - Generalized resolutions of the identity for closed symmetric ordinary differential operators, Proc. Nat. Acad. Sci. U.S.A. vol. 42 (1956) pp. 638-642.

5 . - Generalized resolutions of the identity for symmetric ordinary differential operators, Ann. of Math. vol. 68 (1958) pp. 378-392.

6. E. Hille and R. S. Phillips, Functional analysis and semi-groups, Amer. Math. Soc. Colloquium Publications, vol. 31, 1957.

7. N. Levinson, Transform and inverse transform expansions for singular self-adjoint differential operators, Illinois J. Math. vol. 2 (1958) pp. 224-235.

8. M. A. Naimark, Spectral functions of a symmetric operator, Izv. Akad. Nauk SSSR. Ser. Mat. vol. 4 (1940) pp. 277-318.

9. - Extremal spectral functions of symmetric operators, Izv. Akad. Nauk SSSR. Ser. Mat. vol. 11 (1947) pp. 327-344.

10. A. V. Straus, Generalized resolvents of symmetric operators, Izv. Akad. Nauk SSSR. Ser. Mat. vol. 18 (1954) pp. 51-86.

Princeton University, Princeton, N. J.

University of California, Los Angeles, Calif.

University OF CALIFORNiA, Riverside, Calif. 